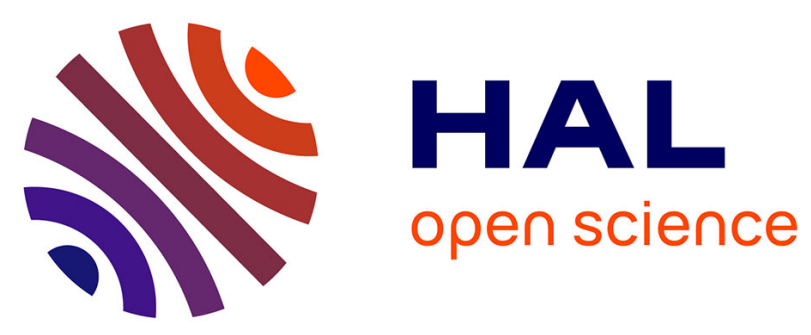

\title{
Milk polar lipids in a high-fat diet can prevent body weight gain: modulated abundance of gut bacteria in relation with fecal loss of specific fatty acids.
}

\author{
Marine Milard, Fabienne Laugerette, Annie Durand, Charline Buisson,
} Emmanuelle Fouilloux-Meugnier, Emmanuelle Loizon, Corinne

Louche-Pélissier, Valerie Sauvinet, Lorna Garnier, Sébastien Viel, et al.

\section{To cite this version:}

Marine Milard, Fabienne Laugerette, Annie Durand, Charline Buisson, Emmanuelle FouillouxMeugnier, et al.. Milk polar lipids in a high-fat diet can prevent body weight gain: modulated abundance of gut bacteria in relation with fecal loss of specific fatty acids.. Molecular Nutrition and Food Research, 2019, 63 (4), pp.1-13. 10.1002/mnfr.201801078 . hal-02008643

\section{HAL Id: hal-02008643 \\ https://univ-lyon1.hal.science/hal-02008643}

Submitted on 11 Feb 2020

HAL is a multi-disciplinary open access archive for the deposit and dissemination of scientific research documents, whether they are published or not. The documents may come from teaching and research institutions in France or abroad, or from public or private research centers.
L'archive ouverte pluridisciplinaire HAL, est destinée au dépôt et à la diffusion de documents scientifiques de niveau recherche, publiés ou non, émanant des établissements d'enseignement et de recherche français ou étrangers, des laboratoires publics ou privés. 


\title{
Milk Polar Lipids in a High-Fat Diet Can Prevent Body Weight Gain: Modulated Abundance of Gut Bacteria in Relation with Fecal Loss of Specific Fatty Acids
}

\author{
Marine Milard, Fabienne Laugerette, Annie Durand, Charline Buisson, \\ Emmanuelle Meugnier, Emmanuelle Loizon, Corinne Louche-Pelissier, Valérie Sauvinet, \\ Lorna Garnier, Sébastien Viel, Karène Bertrand, Florent Joffre, David Cheillan, \\ Lydie Humbert, Dominique Rainteau, Pascale Plaisancié, Laure B. Bindels, \\ Audrey M. Neyrinck, Nathalie M. Delzenne, and Marie-Caroline Michalski*
}

Scope: Enhanced adiposity and metabolic inflammation are major features of obesity associated with altered gut microbiota and intestinal barrier. How these metabolic outcomes can be impacted by milk polar lipids (MPL), naturally containing $25 \%$ of sphingomyelin, is investigated in mice fed a mixed high-fat (HF) diet .

Methods and results: Male C57BI/6 mice receive a HF-diet devoid of MPL (21\% fat, mainly palm oil, in chow), or supplemented with $1.1 \%$ or $1.6 \%$ of MPL (HF-MPL1; HF-MPL2) via a total-lipid extract from butterserum concentrate for 8 weeks. HF-MPL2 mice gain less weight versus HF $(p<0.01)$. Diets do not impact plasma markers of inflammation but in the liver, HF-MPL2 tends to decrease hepatic gene expression of macrophage marker F4/80 versus HF-MPL1 ( $p=0.06$ ). Colonic crypt depth is the maximum in HF-MPL2 $(p<0.05)$. In cecal microbiota, HF-MPL1 increases Bifidobacterium animalis versus HF ( $p<0.05)$. HF-MPL2 decreases Lactobacillus reuteri $(p<0.05)$, which correlates negatively with the fecal loss of milk sphingomyelin-specific fatty acids $(\boldsymbol{p}<0.05)$.

Conclusion: In mice fed a mixed HF diet, MPL can limit HF-induced body weight gain and modulate gut physiology and the abundance in microbiota of bacteria of metabolic interest. This supports further exploration of how residual unabsorbed lipids reaching the colon can impact HF-induced metabolic disorders.

M. Milard, Dr. F. Laugerette, A. Durand, C. Buisson, Dr. D. Cheillan,

Dr. P. Plaisancié, Dr. M.-C. Michalski

Univ Lyon

CarMeN Laboratory

INSERM U 1060

INRA U 1397

INSA Lyon

Université Claude Bernard Lyon 1

69621, Villeurbanne, France

E-mail: marie-caroline.michalski@inra.fr

Dr. E. Meugnier, E. Loizon

Univ Lyon

CarMeN Laboratory

INSERM

INRA

INSA Lyon

Université Claude Bernard Lyon 1

69600, Oullins, France

C. Louche-Pelissier, V. Sauvinet

Centre de Recherche en Nutrition Humaine (CRNH) Rhône-Alpes Centre Européen Pour la Nutrition et la Santé

The ORCID identification number(s) for the author(s) of this article can be found under https://doi.org/10.1002/mnfr.201801078
Centre Hospitalier Lyon Sud

Université Claude Bernard Lyon 1

INSERM, Hospices Civils de Lyon, F-69310, Pierre Bénite, France

L. Garnier, Dr. S. Viel

Laboratoire d'Immunologie

Hospices Civils de Lyon

Centre Hospitalier Lyon Sud

Pierre-Bénite, France

K. Bertrand, Dr. F. Joffre

ITERG-ENMS

Université de Bordeaux

Talence, France

Dr. D. Cheillan

Service Biochimie et Biologie Moléculaire Grand Est

Centre de Biologie Est

Hospices Civils de Lyon

Lyon, France

L. Humbert, Dr. D. Rainteau

Sorbonne Universités

UPMC Univ. Paris 06

École normale supérieure

PSL Research University

CNRS, INSERM, APHP, Laboratoire des Biomolécules (LBM), 27 rue de

Chaligny, Paris, 75005, France

DOI: 10.1002/mnfr.201801078 


\section{Introduction}

Dietary fat intake is mainly provided in the form of $95-97 \%$ of triglycerides $(\mathrm{TG})^{[1,2]}$ and 3-5\% of polar lipids/phospholipids (PL), ${ }^{[3]}$ including glycerophospholipids and sphingolipids. PL are important for their role in food texture and emulsion stability. There is a growing interest for the specific nutritional properties of PL, which could lead to novel functional ingredients. The dietary intake of PL (vegetal and animal sources) represents $2-8 \mathrm{~g}$ of intake per day while sphingolipids represent around 0.3 to $0.4 \mathrm{~g}$ per day. ${ }^{[4,5]}$ Sphingolipids can be categorized as "functional ingredients" because they have structural regulatory functions. Among sphingolipids, sphingomyelin (SM) is involved in the structure of cell membranes, and is present in animal food products such as eggs but in greater amounts in the milk and dairy products. Mammalian milk fat is indeed structured as milk fat globules characterized by a TG core $(\approx 98 \%$ of total lipids) surrounded by the milk fat globule membrane (MFGM), a natural biological membrane whose lipids are thus mainly PL with a unique SM content ( $25 \%$ of MFGM-PL). In rodents, dietary milk polar lipids (MPL) have been shown to reduce the lipid accumulation in the liver, ${ }^{[6]}$ intestinal cholesterol absorption, ${ }^{[7]}$ modulate the postprandial lipid metabolism ${ }^{[8]}$ and are protective against colon carcinogenesis. ${ }^{\left[{ }^{[-11]}\right.}$ It has otherwise been shown in rodents that dietary lipids can impact intestinal barrier and inflammation. Intestinal permeability and barrier function are essentially regulated by a physical (including intestinal epithelial cells and tight junction proteins), a biological (gut microbiota), a chemical (mucus producing cells), and an immunological barrier. Long-chain fatty acid (FA) ${ }^{[12]}$ or a high-fat (HF) diet ${ }^{[13,14]}$ have been shown to impair the tight junction protein expression. Furthermore, it was shown that obesity ${ }^{[15]}$ and metabolic diseases ${ }^{[16]}$ are linked to increased intestinal permeability and translocation of bacteria or bacterial products like endotoxins (LPS) from the intestine to the other tissues. ${ }^{[17,18]}$ Indeed, endotoxins present in the intestinal tract can be found in the circulation after a paracellular passage or co-absorption with dietary lipids. ${ }^{[14,19]}$ Norris et al. have shown that mice fed with a HF diet with milk-SM had lower endotoxemia compared to control HF diet. ${ }^{[20,21]}$ In this context, we must highlight that the intestine is permanently colonized by a rich community of microorganisms, the intestinal microbiota, and in the absence of hypoxia or chemically or heat-induced stressors, a "healthy" microbiota is one component of a proper intestinal barrier.

The aim of our study was to test in mice the hypothesis that incorporation of MPL in a HF diet could impact microbiota profile and gut barrier functions. To advance the state of the art, we tested increasing doses of MPL: i) $1.1 \%$ (i.e., $0.25 \% \mathrm{SM}$ as previously reported in the literature) and ii) $1.6 \%$ (i.e., $0.38 \% \mathrm{SM}$ ) with an aim to perform a benefit/risk assessment of using a higher albeit still realistic dose. Those changes will be interpreted in regard to the metabolic effects on adiposity, gut barrier markers,

Dr. L. B. Bindels, Dr. A. M. Neyrinck, Prof. N. M. Delzenne

Louvain Drug Research Institute

Metabolism and Nutrition Research Group

Université catholique de Louvain

Brussels, Belgium gut microbiota, macrophage markers in liver and adipose tissue, and to the fate of ingested lipids in the gut.

\section{Experimental Section}

\subsection{MPL Ingredient and Diet Composition}

The butterserum concentrate was provided by Corman (Limbourg, Belgium) and the MPL-rich lipid ingredient was obtained therefrom using Folch extraction at ITERG (Fats, Oils and Crops Interbranch Technical Institute, Pessac, France). MPL-rich ingredient contained $63.2 \pm 0.7 \%$ of PL (whose $23.1 \pm 0.5 \%$ of SM, mean \pm SD of duplicate analysis), the balance consisting of TG. It also contained cholesterol due to the natural composition of MFGM lipids. Palm oil was from Alva Food (Rezé, France) and anhydrous milk fat was from Sodiaal-Candia (Paris, France). All the diets were prepared by SAFE (Augy, France). The lipid sources listed in Table 1 were carefully mixed and then incorporated into other components during the manufacturing of the A04 diet at SAFE facility. MPL-rich ingredient was added into HF diet $(21 \%$ of palm oil in chow) at $1.9 \%$ or $3.8 \%$. The specific strategy was to add MPL in the diet and maintain other lipids constant (notably palm oil), thereby increasing total lipids in the diet, rather than attempting to keep total lipids constant. Indeed, it was thought that decreasing palm oil content in the diet could bias the results. Anhydrous milk fat was added in the HF and HF-MPL1 for equal milk TG content among diets. This way, the three high-fat groups differed only by the amount of MPL. The final composition of the diets and of MPL-rich ingredient are shown in Table 1; HF-MPL1 diet contained 1.1\% of MPL and HF-MPL2 contained $1.6 \%$ of MPL. We also analyzed the FA composition (Table S1, Supporting Information) and the SM profile (Table S2, Supporting Information). The SM profile was analyzed by ESI-MS/MS. Briefly, extraction from $50 \mathrm{mg}$ of diet pellets and analyses were realized as previously described. ${ }^{[22]}$

\subsection{Animals and Dosage Information/Dosage Regimen}

Male C57Bl/6 mice (8-week-old at the beginning of the experiment; Janvier Laboratories, France) were housed in groups of five per cage in a controlled environment $\left(24 \pm 1{ }^{\circ} \mathrm{C}, 12 \mathrm{~h}\right.$ daylight cycle, free access to food and water). After 2 weeks of acclimatization with a chow diet, they were randomly divided into three groups of 15 mice and were then fed experimental diets for 8 weeks in which different lipids were mixed within chow (Table 1): the control group (HF) fed an HF diet devoid of MPL (21\% palm oil $+1.4 \%$ of anhydrous milk fat), the HF-MPL1 group ( $21 \%$ palm oil $+0.7 \%$ of anhydrous milk fat $+1.9 \%$ of MPL-rich ingredient), and the group HF-MPL2 (21\% palm oil $+3.8 \%$ of MPL-rich ingredient). This way, all high-fat diets contained the same amount of milk TG (1.4\%) but differed by the amount of MPL ( $0 \%, 1.1 \%$, or $1.6 \%$ as specifically analyzed). Of note, $1.1 \%$ of MPL is equivalent to $\approx 25 \mathrm{mg} \mathrm{SM} \mathrm{kg}-1$ of body weight in a human ${ }^{[20,23]}$ using the Human Equivalent Dose calculation of the FDA. ${ }^{[2]}$ An additional group was fed a chow diet (low-fat, LF) to be used as a reference group. Body weight and food intake were 
Table 1. Ingredients proportion and lipid analysis of experimental diets.

\begin{tabular}{|c|c|c|c|c|}
\hline Ingredients (g per $100 \mathrm{~g}$ diet) & LF & $\mathrm{HF}$ & HF-MPL1 & HF-MPL2 \\
\hline \multicolumn{5}{|c|}{ Components used in formulation } \\
\hline A04 SAFE: & 100 & 77.6 & 76.4 & 75.2 \\
\hline Nitrogen-free extract & 60.4 & 46.9 & 46.1 & 45.4 \\
\hline incl. starch & 33.8 & 33.8 & 33.2 & 32.7 \\
\hline incl. sugars & 3.2 & 2.5 & 2.4 & 2.4 \\
\hline Proteins & 16.1 & 12.5 & 12.3 & 12.1 \\
\hline Fibers & 3.9 & 3.0 & 3.0 & 2.9 \\
\hline Minerals Ash & 4.6 & 3.6 & 3.5 & 3.5 \\
\hline Moisture & 11.9 & 9.2 & 9.1 & 8.9 \\
\hline Lipids & 3.1 & 2.4 & 2.4 & 2.3 \\
\hline Lipids added: & - & 22.4 & 23.6 & 24.8 \\
\hline Palm oil & - & 21 & 21 & 21 \\
\hline Anhydrous milk fat (TG) & - & 1.4 & 0.7 & - \\
\hline MPL ingredient & - & - & 1.9 & 3.8 \\
\hline incl. TG & - & - & 0.7 & 1.4 \\
\hline \multicolumn{5}{|c|}{ Lipid composition of diets after analysis } \\
\hline Phospholipids: & 0.27 & 0.16 & 1.1 & 1.6 \\
\hline PE (29\% of PL) & 0.02 & 0.01 & 0.23 & 0.36 \\
\hline $\mathrm{PI}(7 \%$ of $\mathrm{PL})$ & 0.03 & 0.02 & 0.07 & 0.07 \\
\hline LPE+PS ( $10.1 \%$ of PL) & 0.05 & 0.02 & 0.16 & 0.25 \\
\hline PC $(30.8 \%$ of $P L)$ & 0.1 & 0.06 & 0.35 & 0.45 \\
\hline SM $(23.1 \%$ of $P L)$ & $<0.007$ & 0.01 & 0.25 & 0.38 \\
\hline LPC & 0.06 & 0.04 & 0.06 & 0.08 \\
\hline \multicolumn{5}{|l|}{ Sterols: } \\
\hline Cholesterol & 0.007 & 0.010 & 0.030 & 0.036 \\
\hline Phytosterols & 0.067 & 0.069 & 0.066 & 0.063 \\
\hline Total fat & 3.2 & 22.7 & 24.6 & 24.8 \\
\hline \multicolumn{5}{|c|}{ Energy content after diet fat content analysis (\%energy) } \\
\hline Proteins & 19.3 & 11.5 & 10.9 & 10.8 \\
\hline Nitrogen-free extract & 72.4 & 43.0 & 40.9 & 40.7 \\
\hline incl. starch + sugars & 55.9 & 33.2 & 31.6 & 31.5 \\
\hline Lipids & 8.4 & 45.6 & 48.2 & 48.5 \\
\hline
\end{tabular}

HF, high-fat; LF, low-fat; LPC, lysophosphatidylcholine; LPE, lysophosphatidylethanolamine; MPL, milk polar lipids ; PC, phosphatidylcholine; PE, phosphatidylethanolamine; PI, phosphatidylinositol; PL, phospholipids; PS, phosphatidylserine; SM, sphingomyelin; TC, triglycerides.

recorded twice per week. After 8 weeks of dietary treatment and a night period of fasting, mice were anesthetized with isoflurane before exsanguination and tissue sampling. After laparotomy, blood was collected by cardiac puncture with heparinized syringes. Duodenum, jejunum, colon, caecum, liver, and adipose tissue (epididymal and subcutaneous) were harvested, immediately frozen in liquid nitrogen and stored at $-80{ }^{\circ} \mathrm{C}$. Other samples of intestine and liver were collected for immunohistochemistry. All procedures were approved by the local ethical committee for animal experimentation of Lyon 1 University CEEA-55 (CNREEA 2012) (project \#DR2017-14-v2, evaluation \#2017021414372273_v3).

\subsection{Plasma Biochemistry}

Plasma was collected by centrifugation $\left(2000 \times \mathrm{g}, 5 \mathrm{~min}, 4^{\circ} \mathrm{C}\right)$. Endotoxemia was determined using the limule amoebocyte lysate assay (Associates of Cape Cod, MA, USA) as previously described. ${ }^{[25]}$ Concentration of the lipopolysaccharide-binding protein (LBP) was detected via ELISA kit (Hycult Biotech Inc., Plymouth Meeting, PA, USA) and detection of soluble cluster of differentiation 14 (sCD14) was done using ELISA kits from R\&D Systems (Minneapolis, MN, USA). Plasma concentration of IL-6, tumor necrosis factor $\alpha$ (TNF- $\alpha$ ), IL-1 $\beta$, IL-10, monocyte chemoattractant protein 1 (MCP-1), chemokine ligand CXCL1 (KC) (limit of detection: $6 \mathrm{pg} \mathrm{mL}^{-1}$ ) and CXCL2 (MIP-2) (limit of detection: $160 \mathrm{pg} \mathrm{mL}^{-1}$ ) were determined using a multiplex immunoassay kit (Milliplex MAP Mouse Cytokine, Mcytomag-70K, Merck Millipore, Fontenay sous Bois, France) and measured using a Bio-plex 200 systems (Biorad, CA, USA).

\subsection{Gut Permeability Analysis}

Intestinal permeability was examined using a low-molecular weight (4-kDa) FITC-dextran (Sigma-Aldrich, Saint-Quentin Fallavier, France). After 8 weeks of diet, mice were fasted overnight and FITC-dextran was administered by oral gavage (0.6 $\mathrm{mg} \mathrm{g}^{-1}$ body weight; $125 \mu \mathrm{g} \mu \mathrm{L}^{-1}$ solution). After $4 \mathrm{~h}$, plasma was collected by centrifugation and kept in dark. FITC levels were determined using a fluorescence spectrophotometer (SAFAS Xenius XL, SAFAS Monaco, France) at excitation $485 \mathrm{~nm}$ and emission $533 \mathrm{~nm}$.

\subsection{Tissue RNA Extraction and Quantitative Reverse Transcriptase-PCR}

Total RNA was extracted from whole intestine segments, liver, and epididymal/subcutaneous adipose tissue (EAT/SAT) with TRI Reageant (Sigma, Saint-Quentin-Fallavier, France). RNA concentration was measured with Multiskan GO microplate spectrophotometer (Thermo Fisher Scientific, Waltham, MA, USA) and samples with A260/280 ration between 1.7 and 2.1 were considered of good purity. Reverse transcription (RT) was performed using PrimeScript RT reagent kit (Ozyme, Saint Quentin en Yvelines, France) with $1 \mu \mathrm{g}$ of RNA. RT-qPCR (quantitative PCR) assays were performed using a Rotor-Gene Q (Qiagen, Hilden, Germant) and SYBR qPCR Premix Ex Taq (Tli RNaseH Plus) reagents. The list of the PCR primers used is shown in Table S3, Supporting Information. Tata-box-binding protein (TBP) expression was used as internal standard (IS) for normalization of target mRNA expression.

\subsection{Analysis of the Fatty Acids from Triglycerides and Phospholipids in the Liver and of Total Fatty Acids in the Feces}

Approximately $100 \mathrm{mg}$ cryogenically crushed liver sample was weighed before proceeding to the total lipid extraction using a mixture of chloroform/methanol (2:1, v/v) according to the 
Folch method. ${ }^{[26]}$ ISs, TG-C17:0 and PC-C17:0, were added. The TG and the PL fractions were then obtained from one-fifth of the total lipid extract by thin-layer chromatography on silica-gel plates with a mobile phase of hexane:diethyl ether:acetic acid $(80: 20: 1, \mathrm{v} / \mathrm{v} / \mathrm{v})$ and were submitted after drying to direct methylation according to a modification of the technique described elsewhere. ${ }^{[2]}$ Briefly both fractions were dissolved in a solvent mixture of methanol/hexane $(4: 1 \mathrm{v} / \mathrm{v})$ and $200 \mu \mathrm{L}$ of acetyl chloride. The tubes were tightly closed and subjected to methanolysis at $100{ }^{\circ} \mathrm{C}$ for $1 \mathrm{~h}$. After cooling the tubes in ice, an $11 \% \mathrm{~K}_{2} \mathrm{CO}_{3}$ solution was added and the phases were vortexed. Then hexane was added and after separation, the organic layer was isolated and dried under a stream of nitrogen. The Fatty Acid Methyl Esters (FAMEs) of the TG and PL fractions were reconstituted in hexane. Feces were dried under a stream of nitrogen before being crushed in a stainless-steel mortar using a pestle. Approximately $5 \mathrm{mg}$ were weighed before proceeding to two successive lipid extractions using a mixture of chloroform/methanol (1:1, v/v). The heptadecanoic acid, an IS, was added before lipid extraction. Organic phase was collected after centrifugation $\left(10 \mathrm{~min}, 4^{\circ} \mathrm{C}, 2608\right.$ g). Total lipid extract was dried and submitted to direct methylation as above. The amounts of FA were measured by GC-EIMS (EI, electron ionization) using a quadrupole mass spectrometer (model MS 5975, Agilent Technologies, Massy, France) connected to a gas chromatograph (model GC6890, Agilent Technologies). It was equipped with a fused-silica column (SP2380, $60 \mathrm{~m} \times 0.20 \mathrm{~mm} \times 0.25 \mu \mathrm{m}$ film thickness; Supelco). Helium was used as the carrier gas. Injection was performed in splitless mode at $240{ }^{\circ} \mathrm{C}$. FA were separated with the following oven program: i) $50^{\circ} \mathrm{C}$ for $1 \mathrm{~min}$; ii) increase at a rate of $20^{\circ} \mathrm{C} \mathrm{min}{ }^{-1}$ to $175^{\circ} \mathrm{C}$ and hold for $9.75 \mathrm{~min}$; iii) increase at a rate of $2^{\circ} \mathrm{C} \mathrm{min}{ }^{-1}$ to $217^{\circ} \mathrm{C}$ and hold at $217^{\circ} \mathrm{C}$ for $1 \mathrm{~min}$. Mass spectra were obtained from the total ion chromatogram over a mass range of $\mathrm{m} / z$ 35-450.

\subsection{Gut Microbiota Analyses of the Cecal Content}

At the end of the experiment, the total cecum was collected and weighed before storage at $-80^{\circ} \mathrm{C}$. Cecum content was collected and weighed before extraction. The gut microbiota composition was assessed by 16S rRNA gene analyses using qPCR. Genomic DNA was extracted from the cecal content using a QIAamp DNA stool Mini Kit (Qiagen, Hilden, Germany), including a beadbeating step (glass bead $0.45 \mu \mathrm{m}$, VWR, Belgium), according to manufacturer's instructions. qPCR was performed with primers and annealing temperature described in Table S4, Supporting Information. The qPCR was performed with a StepOne plus RealTime PCR system and software (Applied Biosystems, Den Ijssel, The Neverlands) using SYBR Green (Eurogentec, Verviers, Belgium) for detection. All samples were run in duplicate in a single 96-well reaction plate. A standard curve (performed in duplicate) was included on each qPCR plate by diluting genomic DNA from pure culture (fivefold serial dilution). Cell counts were determined by plating and expressed as "colony-forming unit" (CFU) before DNA isolation. For Lactobacillus reuteri, standard DNA was quantified based on L. acidophilus DNA (DSMZ, Braunshweig, Germany). ${ }^{[28]}$

\subsection{Immunohistochemistry of Liver}

Imaging experiments were performed on the CellimaP platform in Dijon. Liver sections were fixed in $4.5 \%$ formalin containing fixation solution (Roti Histofix, Carl Roth, Karlsruhe, Germany). After $48 \mathrm{~h}$, samples were transferred in $70 \%$ ethanol until paraffin inclusion. Serial paraffin sections $(4 \mu \mathrm{m})$ were rehydrated and saturated. Immunohistochemical staining was realized using monoclonal primary antibody against F4/80 (anti-mouse produced in rat) (diluted 1:200, Abcam, Cambridge, UK) during $1 \mathrm{~h}$. As a control, slides were incubated without primary antibody. After PBS washing, sections were incubated with secondary antibody antirat produced in rabbit (Vector Laboratories, CA, USA) during $1 \mathrm{~h}$ and to finished, anti-rabbit horseradish peroxidase-labeled (Dako Diagnostics, Agilent Technologies, Edinburgh, UK) for $30 \mathrm{~min}$. Sections were counterstained with Mayer's hematoxylin, deshydrated, cleared in xylene. Slides were mounted with dibutyl phthalate xylene and dried overnight before examination. Liver histology was examined using the light microscope at $20 \times$ magnification and quantification was performed by TissueIA software, Leica Microsystems.

\subsection{Quantitative Analysis of Intestinal Goblet Cells and Colonic Crypt Depth}

Imaging experiments were performed on the CellimaP platform in Dijon. The PFA-fixed intestines were embedded in paraffin and $4 \mu \mathrm{m}$ sections were prepared. Proximal colon was then stained with hematoxylin and eosin to quantified length of villus and depth of crypts. Colonic crypts depths were performed and defined as the depth of the invagination between two villi. Crypt depths were measured with TissueIA software in ten well-oriented crypt-villus units (two cross sections for each mice). To count the number of goblet cells, sections were stained with alcian blue and periodic acid-Schiff's reagent to visualize the total mucins. Goblet cells were counted for villus-crypt axis of colon.

\subsection{Measurement of Bile Acid Molecular Species}

Bile acids measurements were performed on mouse bile by HPLC-MS/MS as described in Humbert et al. ${ }^{[29]}$ For the HPLCMS/MS analysis, the separation of bile acids as a function of polarity was accomplished using an analytical column (Pinnacle II C18, Restek, Lisses, France) fitted on an HPLC binary pump (Agilent 1100, Agilent Technologies France, Massy, France). Briefly, an IS was added to the samples before extraction (23-nor-5 $\beta$-cholanoic acid-3 $\alpha, 12 \alpha$-diol at $1 \mathrm{mg} \mathrm{mL}^{-1}$ ). The preanalysis cleanup procedure of $50 \mu \mathrm{L}$ plasma was achieved by protein precipitation ( $200 \mu \mathrm{L}$ acetonitrile containing IS) and centrifugation $(12000 \times \mathrm{g})$. Supernatant diluted in ten volumes of deionized water were loaded on SPE C18 Chromabond Cartidges (Macherey-Nagel, Düren, Germany), and after washes, elution was performed with methanol. After separation in an analytical column (Pinnacle II C18), ESI was performed in negative mode. Each peak was identified by comparing the spectrum of a range including species of bile acids. For quantitation, data were 
A

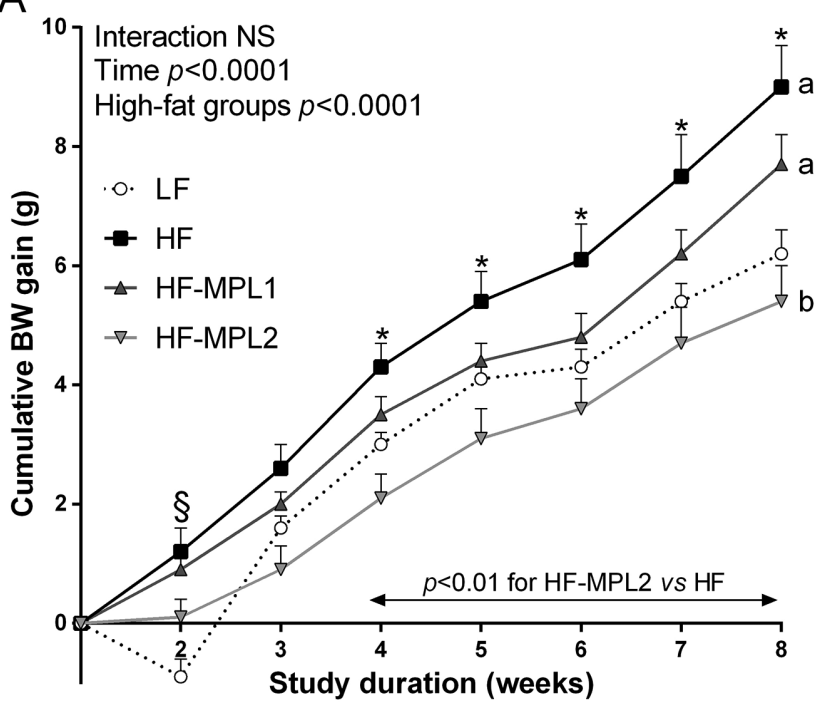

B
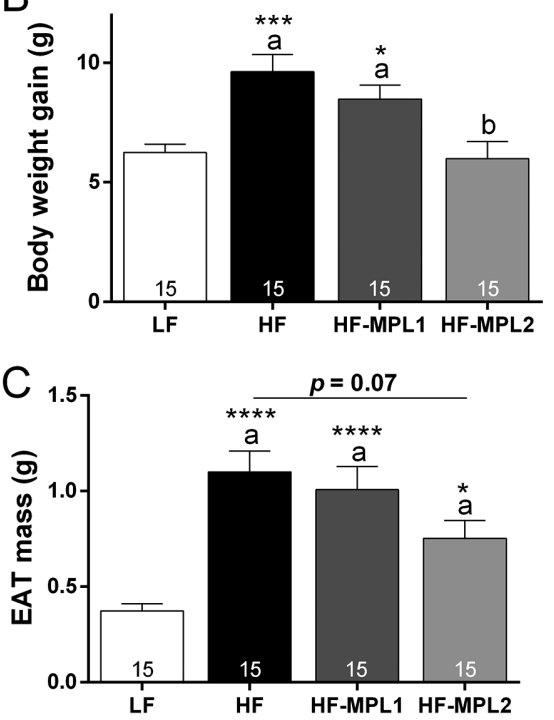

Figure 1. MPL2 supplementation in high fat diet reduced body weight gain. Mice were submitted to a chow-based high-fat diet (HF) or a high-fat diet supplemented with MPL (1.1\% or 1.6\%) during 8 weeks. A) Cumulative body weight (BW) gain evolution. Two-way ANOVA between HF groups were used, testing HF group effect, time effect, and interaction (HF groups not sharing a common letter were significantly different at $p<0.01$ for both 7 and 8 weeks). The mean of each HF condition was compared with a LF reference group (ANOVA followed by Dunnett's post hoc test; * $p<0.05 \mathrm{HF}$ versus LF; $\mathbb{I}_{p}<0.05 \mathrm{LF}$ versus HF and HF-MPL1). B) BW gain and C) EAT weight at the end of dietary intervention. To test the impact of MPL amount in the $\mathrm{HF}$ diet, data of the three HF groups were analyzed by one-way ANOVA (values not sharing a common superscript letters were significantly different). Additionally, the mean of each HF condition was compared with a LF reference group to evaluate HF induced alterations (" $p<0.05$ ANOVA followed by Dunnett's post hoc test HF vs LF). Results represent mean \pm SEM. Number of mice in each group is indicated within the bars ( $n=15$ mice).

acquired using Analyst V.1.4.2 software. The bile acid quantitation was expressed as the percentage of each specific bile acid $( \pm$ SEM) out of the total bile acids after calibration of the method, with weighed mixtures and normalization relative to the IS.The hydrophobicity index reflects bile acids hydrophobicity, taking into account the retention time of different bile acids on a C18 column with a gradient of methanol; the lithocolic acid (LCA) has the highest retention time, the taurosodeoxycholic acid (TUDCA-3S) has the lowest.

\subsection{Statistical Analysis}

All data, presented as mean \pm SEM, were analyzed with Graph Pad Prism Software (version 7.0, San Diego, CA, USA). Normality of data was examined using Shapiro-Wilk test. To test the hypothesis that the amount of MPL within the HF diet modified the final outcome of the HF diet, the data of the three HF groups were analyzed by a one-way analysis of variance (ANOVA) followed by a Tukey's post hoc multiple comparisons test on normal data. For nonnormal data, a Kruskal-Wallis test was performed followed by a Dunn post hoc test. In figures and tables, values not sharing a common letter were significantly different among high-fat groups. Additionally, to check whether each HF group induced alteration compared with a low-fat reference group (chow) after 8 weeks of diet, a one-way ANOVA followed by a Dunnett post-hoc test was performed to assess statistical differences with the LF group (an asterisk represents a significant difference of a highfat group with LF group, one hashtag is used when all high-fat groups differed from the low-fat group). To analyze differences in cumulative body weight gain and energy intake $\left(\mathrm{kJ} \mathrm{d}^{-1}\right)$ between HF groups during the 8 weeks of intervention, two-way ANOVA between HF groups was used. As the interaction term (time $\times$ HF group) was not significant, this analysis was followed by Bonferroni post hoc multiple comparisons test (HF groups not sharing a common letter were significantly different for both 7 and 8 weeks). Additionally, the mean of each high-fat condition during the 8 weeks of intervention was compared with the LF reference group using two-way ANOVA followed by Dunnett post-hoc test; if the interaction term was significant, an unpaired test was performed. Altogether throughout the manuscript, letters refer to comparisons between HF groups (primary hypothesis tested: metabolic impact of MPL addition in an HF diet) while symbols refer to comparisons between each HF group and the LF group (to assess HF induced metabolic alterations). To evaluate possible relationships among the various outcomes, Spearman correlations were performed using Graph Pad Prism Software. Differences with $p<0.05$ were considered significant.

\section{Results}

\subsection{HF-MPL2 Limits HF-Induced Body Weight Gain and Epididymal Fat Mass}

Body weight and EAT weight increased in HF group compared with LF reference group $(p<0.001)$. After 8 weeks, compared to HF mice, HF-MPL2 mice gained significantly less weight than did HF mice (by $\approx 37.8 \%, p<0.01$ ) and the weight of the EAT was nonsignificantly reduced (by $\approx 23.3 \%, p=0.07$ ) (Figure 1 ). 
Table 2. Mice metabolic parameters and plasma concentration of inflammatory markers.

\begin{tabular}{|c|c|c|c|c|}
\hline & LF & $\mathrm{HF}$ & HF-MPL 1 & HF-MPL2 \\
\hline \multicolumn{5}{|l|}{ Metabolic parameters } \\
\hline Initial body weight (g) & $22.9 \pm 0.35$ & $23.8 \pm 0.5$ & $23.1 \pm 0.3$ & $23.0 \pm 0.2$ \\
\hline Final body weight (g) & $29.8 \pm 0.6$ & $32.8 \pm 0.8^{\mathrm{a}, *}$ & $30.8 \pm 0.6^{b}$ & $28.4 \pm 0.6^{c}$ \\
\hline Food intake $\left(k J d^{-1}\right)$ & $61.9 \pm 1.4^{\#}$ & $79.7 \pm 1.7$ & $76.9 \pm 4.2$ & $83.0 \pm 1.7$ \\
\hline Liver weight (g) & $1.14 \pm 0.03$ & $1.05 \pm 0.03$ & $1.05 \pm 0.03$ & $0.99 \pm 0.03^{\text {竍 }}$ \\
\hline \multicolumn{5}{|l|}{ Plasma concentrations } \\
\hline MCP-1 (pg mL $\left.L^{-1}\right)$ & $81.0 \pm 12.7$ & $59.0 \pm 16.6$ & $56.0 \pm 6.2$ & $45.0 \pm 8.1$ \\
\hline $\mathrm{CXCL} 1\left(\mathrm{pg} \mathrm{mL} \mathrm{L}^{-1}\right)$ & $188.4 \pm 27.3$ & $107.6 \pm 38.8$ & $114.2 \pm 17.2$ & $78.33 \pm 23.2^{\prime \prime}$ \\
\hline $\operatorname{LBP}\left(\mu \mathrm{g} \mathrm{mL}^{-1}\right)$ & $6.1 \pm 0.7$ & $4.9 \pm 0.2$ & $5.2 \pm 0.2$ & $5.4 \pm 0.3$ \\
\hline $\mathrm{sCD} 14\left(\mathrm{ng} \mathrm{mL}^{-1}\right)$ & $70.7 \pm 3.6^{\#}$ & $50.4 \pm 4.1$ & $46.8 \pm 3.4$ & $44.9 \pm 3.4$ \\
\hline
\end{tabular}

Values represent mean \pm SEM. Significant differences between HF groups were analyzed by Kruskal-Wallis test (for nonnormal data) or ANOVA (for normal data): means of $\mathrm{HF}$ groups not sharing a common letter are statistically different; $p<0.05$ $* p<0.05, * * p<0.01$ versus LF group (ANOVA followed by Dunnett test; for table clarity to avoid numerous asterisks when all HF groups were different from the LF reference group: ${ }^{\#} p<0.01 \mathrm{LF}$ vs each HF group).; CXCL1, chemokine ligand 1; HF, high fat; LBP, lipopolysaccharide binding protein; LF, low-fat; MCP-1, Monocyte chimioattractant protein 1; MPL, milk polar lipids; SCD 14, soluble cluster of differentiation

Moreover, HF-MPL2 group was the only one that did not gain more weight than the LF reference group (Figure 1B). Of note, liver, stomach, and cecum weights were not different among HF groups. Average food consumption did not differ among groups (results not shown); average energy intake over 8 weeks was higher for all high-fat groups versus reference LF group (Table 2) as well as along the feeding period (Figure S1, Supporting Information); however, energy intake was not different between HF, HF-MPL1, and HF-MPL2 groups (Table 2, Figure S1, Supporting Information). Among HF groups, a positive correlation was observed between body weight gain and EAT mass $(r=0.86$, $p<0.0001$ ). HF-MPL1 and HF-MPL2 did not modify liver weight compared to HF group but liver weight was lower in HF-MPL2 versus LF reference group (Table 2).

\subsection{Influence of Diets on Macrophage Infiltration Markers and Liver Lipid Content}

We analyzed different circulating transporters and receptors in plasma related to the response to endotoxins (LPS), such as LBP, sCD14, and the concentration of anti- or pro-inflammatory markers (Table 2). Endotoxemia was undetectable and LBP and SCD14 were similar among HF groups, and SCD14 was even lower in HF group versus LF group, suggesting that the intestinal permeability was not altered and no bacterial translocation occurred. Inflammatory cytokines IL- $1 \beta$, TNF- $\alpha$, IL-6, IL-10, and CXCL2 concentrations were undetectable; MCP-1 and CXCL1 plasma concentrations were similar among groups and not different between HF and LF reference group (Table 2).

We then assessed more local impacts in tissues by analyzing treatment effects on different markers of macrophage infiltration in liver and in adipose tissue (Figure 2). Of note, except F4/80 in the liver, markers were altogether not increased in the HF group compared to a LF reference group. The mRNA level of F4/80 tended to be lower in the liver of HF-MPL2 versus HF-MPL1 group $(p=0.06)$. HF-MPL2 group was the only one that did not increase liver F4/80 compared with the LF reference group. Furthermore, we found a significant positive correlation between the gene expression of F4/80 in the liver and its expression in SAT $(r=0.618 ; p<0.01)$. F4/80 immunostaining did not reveal differences between groups (Figure 2B,C). Compared to $\mathrm{HF}$ control, gene expression of other inflammatory markers in liver (IL-6, IL-1 $\beta$, and LBP) was not impacted by MPL (data not shown). In SAT and in EAT, no significant differences were observed among HF groups regarding the gene expression of F4/80 and cluster of differentiation 68 (Cd68), another global marker of macrophage infiltration (Figure 2D,E). Cluster of differentiation $11 \mathrm{c}(\mathrm{Cd} 11 \mathrm{c})$, which is more specific of proinflammatory macrophages, was not differentially expressed in SAT; an increase was observed in EAT but only in HF-MPL1 versus HF group and versus LF group (Figure $2 \mathrm{~F}$ ).

We also tested whether MPL impacted hepatic lipids: liver content in PL and TG were not different among groups (Table 3). In hepatic PL that represent structural lipids, total SFA, MUFA, and PUFA did not differ among groups but HF-MPL2 group was richer than HF group in homolinoleic acid (20:2), tricosylic acid (C23:0), and lignoceric acid (C24:0) that are typical of milk SM (Table 3). In hepatic TG that represent storage lipids, altogether in both HF-MPL1 and HF-MPL2 groups, oleic acid (C18:1) was decreased while palmitoleic acid (C16:1) was increased $(p<0.01)$ compared to HF group. In both hepatic PL and TG, vaccenic acid (18:1 n-7) was lowest in HF-MPL2 group.

\subsection{MPL Supplementation has Only Minor Impact on Gut Barrier but Increases Colonic Crypt Depth}

Minor effects of the treatments on indirect markers gut barrier functions were observed. Indeed, paracellular gut barrier integrity assessed by FITC-dextran test did not reveal significant permeability and did not differ among groups (Figure S2A, Supporting Information). The mRNA levels of some tight-junction proteins were measured; no differences were observed for $\mathrm{Oc}$ cludin and junctional adhesion molecule 1 (Jam-1) in the duodenum (data not shown). Altogether, MPL supplementation significantly increased the mRNA levels of Zonula-occludens 1 (Zo-1) $(p<0.05$ pooled data of HF-MPL1 and HF-MPL2 versus HF; $p=0.06$ for HF-MPL2 versus HF; Figure S2B, Supporting Information). No differences were observed for the gene expression of these tight junction proteins in the jejunum and the colon (data not shown).

The small intestine did not appear to be altered and no differences in crypt depth, villus length, or mucus cell numbers were observed (data not shown). However in the colon, crypt depth was increased in HF-MPL2 group versus HF-MPL1 and versus HF ( $p<0.05$; Figure $3 \mathrm{~A}, \mathrm{~B})$. The structure of the villi and crypts of the colon appeared normal among diets (no major alteration versus LF reference) and mucus cell number remained unaffected among HF groups, despite a lower number in HF-MPL2 versus LF reference group (Figure 3C). We tested the possible involvement of other cell types in increased crypt depth: the colonic mRNA level of proglucagon, which is the 
A

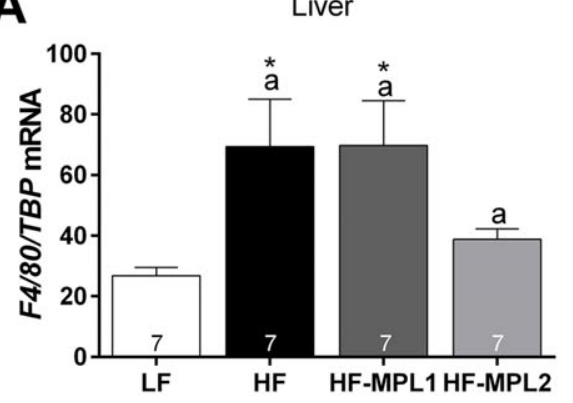

C
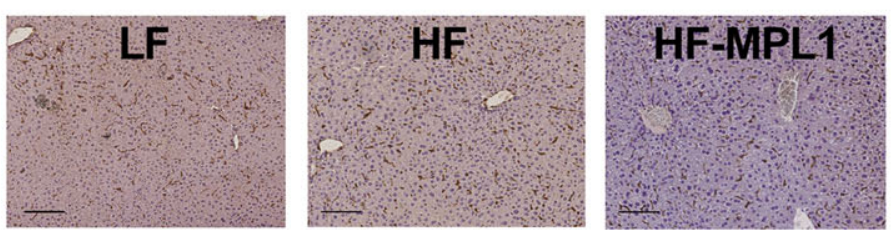

D

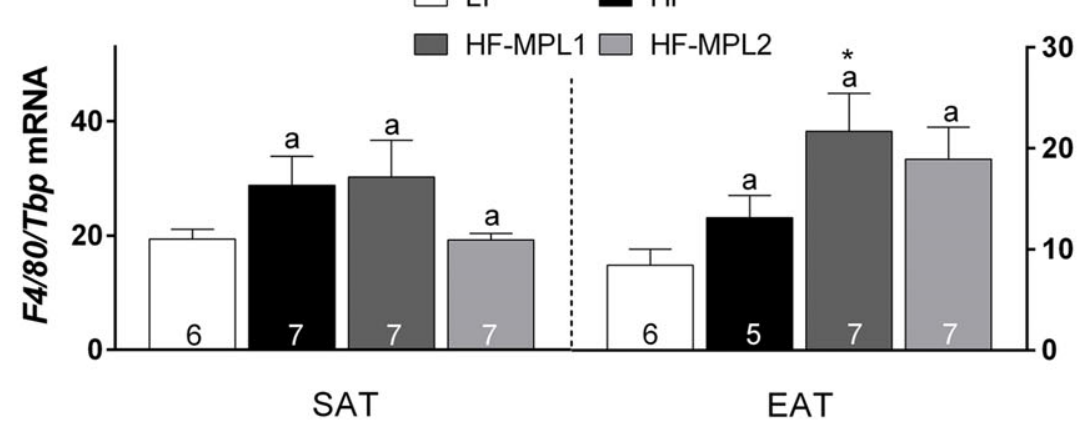

E

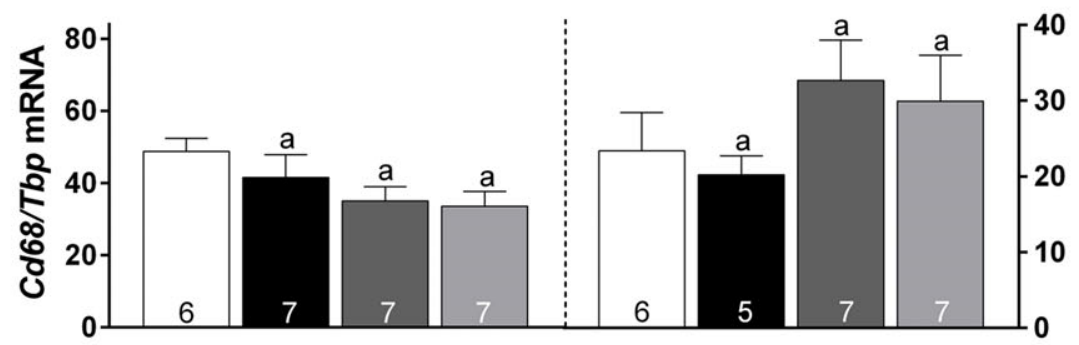

F

SAT

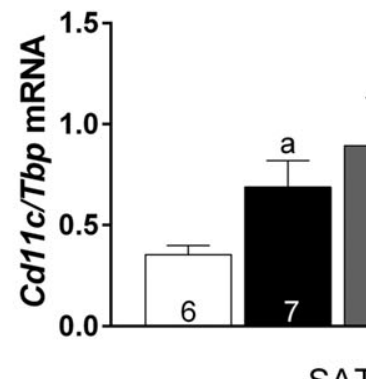

SAT

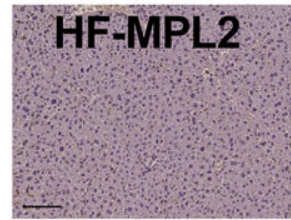

B

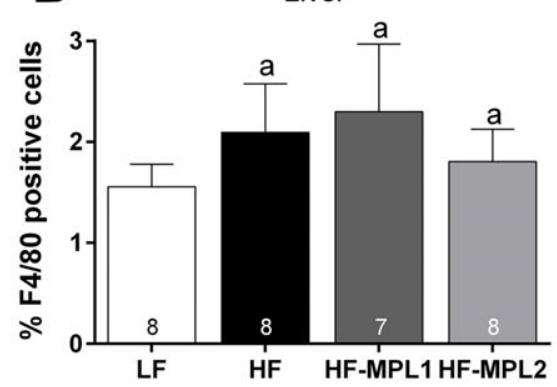

- $\mathrm{HF}$ 
Table 3. Fatty acid content and profile in mice liver.

\begin{tabular}{|c|c|c|c|c|c|c|c|c|c|}
\hline Fatty acids & LF & $\mathrm{HF}$ & HF-MPL1 & HF-MPL2 & Fatty acids & LF & $\mathrm{HF}$ & HF-MPL 1 & HF-MPL2 \\
\hline \multicolumn{5}{|c|}{ Hepatic PL } & \multicolumn{5}{|c|}{ Hepatic TG } \\
\hline Total ( $\mu \mathrm{g} \mathrm{mg}^{-1}$ liver) & $11.4 \pm 0.6$ & $14.0 \pm 1.5$ & $15.5 \pm 1.7$ & $12.3 \pm 0.8$ & Total ( $\mu \mathrm{g} \mathrm{mg}^{-1}$ liver) & $73.0 \pm 0.7$ & $66.8 \pm 7.7$ & $68.0 \pm 8.4$ & $81.6 \pm 5.4$ \\
\hline \multicolumn{5}{|c|}{$\mathrm{Wt} \%$ of total FA } & \multicolumn{5}{|c|}{ Wt $\%$ of total FA } \\
\hline $14: 0$ & $0.10 \pm 0.00^{\#}$ & $0.07 \pm 0.00$ & $0.05 \pm 0.01$ & $0.06 \pm 0.00$ & $12: 0$ & $0.12 \pm 0.01$ & $0.10 \pm 0.01$ & $0.08 \pm 0.01^{\text {放 }}$ & $0.1 \pm 0.01$ \\
\hline 15:0 & $0.09 \pm 0.01^{\#}$ & $0.05 \pm 0.00$ & $0.05 \pm 0.00$ & $0.05 \pm 0.00$ & 14:0 & $1.1 \pm 0.04^{\#}$ & $0.78 \pm 0.04$ & $0.70 \pm 0.03$ & $0.77 \pm 0.02$ \\
\hline $16: 0$ & $23.7 \pm 0.37$ & $24.7 \pm 0.30^{*}$ & $24.3 \pm 0.15$ & $24.2 \pm 0.24$ & $14: 1$ & $0.09 \pm 0.01^{\#}$ & $0.05 \pm 0.00$ & $0.04 \pm 0.00$ & $0.04 \pm 0.00$ \\
\hline $16: 1$ & $1.93 \pm 0.10^{\#}$ & $1.03 \pm 0.07$ & $1.04 \pm 0.02$ & $0.99 \pm 0.03$ & 15:0 & $0.18 \pm 0.01^{\#}$ & $0.12 \pm 0.00^{\mathrm{a}}$ & $0.13 \pm 0.00^{\mathrm{a}, \mathrm{b}}$ & $0.14 \pm 0.00^{b}$ \\
\hline 18:0 & $13.7 \pm 0.36$ & $14.2 \pm 0.29$ & $14.2 \pm 0.18$ & $14.4 \pm 0.17$ & $16: 0$ & $23.8 \pm 0.53^{\#}$ & $29.3 \pm 0.39$ & $29.6 \pm 0.13$ & $28.3 \pm 0.53$ \\
\hline $18: 1$ (n-9) cis & $8.31 \pm 0.09^{\#}$ & $10.5 \pm 0.41$ & $10.0 \pm 0.15$ & $10.2 \pm 0.24$ & $16: 1$ & $4.75 \pm 0.6$ & 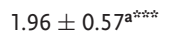 & $5.58 \pm 0.14^{b}$ & $5.53 \pm 0.2^{b}$ \\
\hline 18:1 (n-7) & $2.81 \pm 0.12^{\#}$ & $1.43 \pm 0.04^{\mathrm{a}}$ & $1.38 \pm 0.03^{\mathrm{a}}$ & $1.25 \pm 0.02^{b}$ & $17: 1$ & $0.25 \pm 0.02^{\#}$ & $0.14 \pm 0.01$ & $0.13 \pm 0.01$ & $0.13 \pm 0.00$ \\
\hline $18: 2(n-6)$ cis & $18.6 \pm 0.21^{\#}$ & $14.9 \pm 0.27$ & $14.4 \pm 0.21$ & $14.8 \pm 0.23$ & $18: 0$ & $0.98 \pm 0.11$ & $0.95 \pm 0.05$ & $0.98 \pm 0.05$ & $1.13 \pm 0.06$ \\
\hline $20: 0$ & $0.13 \pm 0.00$ & $0.14 \pm 0.01$ & $0.14 \pm 0.00$ & $0.13 \pm 0.00$ & $18: 1$ (n-9) cis & $30.15 \pm 0.43^{\#}$ & $43.2 \pm 0.57^{\mathrm{a}}$ & $40.74 \pm 0.2^{b}$ & $41.1 \pm 0.51^{b}$ \\
\hline $18: 3(n-6)$ & $0.37 \pm 0.02$ & $0.45 \pm 0.03$ & $0.40 \pm 0.02$ & $0.38 \pm 0.01$ & $18: 1$ (n-7) & $2.96 \pm 0.06^{\#}$ & $1.49 \pm 0.05^{a}$ & $1.44 \pm 0.07^{\mathrm{a}, \mathrm{b}}$ & $1.34 \pm 0.04^{b}$ \\
\hline 20:1 & $0.22 \pm 0.01^{\#}$ & $0.16 \pm 0.01$ & $0.18 \pm 0.00$ & $0.18 \pm 0.01$ & $18: 2(n-6)$ cis & $28.37 \pm 0.75^{\#}$ & $17.6 \pm 0.27^{\mathrm{a}}$ & $16.7 \pm 0.16^{b}$ & $17.3 \pm 0.24^{\mathrm{a}, \mathrm{b}}$ \\
\hline $20: 2$ & $0.13 \pm 0.00$ & $0.11 \pm 0.00^{\mathrm{a}^{*}}$ & $0.13 \pm 0.00^{\mathrm{a}, \mathrm{b}}$ & $0.13 \pm 0.00^{\mathrm{b}}$ & $20: 0$ & $0.07 \pm 0.01$ & $0.06 \pm 0.01$ & $0.06 \pm 0.00$ & $0.06 \pm 0.00$ \\
\hline 22:0 & $0.41 \pm 0.01^{\#}$ & $0.52 \pm 0.03$ & $0.60 \pm 0.03$ & $0.58 \pm 0.02$ & $18: 3(n-6)$ & $1.19 \pm 0.04$ & $1.14 \pm 0.08$ & $0.99 \pm 0.05^{*}$ & $0.97 \pm 0.04^{*}$ \\
\hline $20: 3(n-3)$ & $0.65 \pm 0.04$ & $0.89 \pm 0.07^{*}$ & $0.88 \pm 0.05^{*}$ & $0.83 \pm 0.05$ & $18: 3(n-3)$ & $1.11 \pm 0.05^{\#}$ & $0.37 \pm 0.02$ & $0.35 \pm 0.02$ & $0.38 \pm 0.02$ \\
\hline $20: 4(n-6)$ & $16.7 \pm 0.14^{\#}$ & $18.8 \pm 0.35$ & $18.7 \pm 0.11$ & $18.8 \pm 0.15$ & $20: 1$ & $0.81 \pm 0.08^{\#}$ & $0.36 \pm 0.02$ & $0.33 \pm 0.01$ & $0.36 \pm 0.01$ \\
\hline 23:0 & $0.11 \pm 0.01$ & $0.12 \pm 0.01^{\mathrm{a}}$ & $0.24 \pm 0.01^{\mathrm{a}, \mathrm{b}^{\mathrm{m}}}$ & $0.33 \pm 0.01^{b * t}$ & $20: 2$ & $0.17 \pm 0.01^{\#}$ & $0.09 \pm 0.01$ & $0.06 \pm 0.00$ & $0.06 \pm 0.00$ \\
\hline $20: 5$ & $0.18 \pm 0.01^{\#}$ & $0.11 \pm 0.01$ & $0.12 \pm 0.01$ & $0.12 \pm 0.00$ & $20: 3(n-3)$ & $0.27 \pm 0.01^{\#}$ & $0.19 \pm 0.02$ & $0.17 \pm 0.01$ & $0.18 \pm 0.01$ \\
\hline $24: 0$ & $0.32 \pm 0.01^{\#}$ & $0.28 \pm 0.01^{\mathrm{a}}$ & $0.36 \pm 0.01^{b}$ & $0.37 \pm 0.01^{\mathrm{b}}$ & $20: 4(n-6)$ & $1.39 \pm 0.08^{\#}$ & $1.0 \pm 0.05$ & $0.94 \pm 0.03$ & $0.97 \pm 0.04$ \\
\hline $24: 1$ & $0.54 \pm 0.06^{\#}$ & $0.42 \pm 0.01$ & $0.43 \pm 0.01$ & $0.43 \pm 0.01$ & $20: 5$ & $0.23 \pm 0.01^{\#}$ & $0.1 \pm 0.01$ & $0.11 \pm 0.01$ & $0.11 \pm 0.01$ \\
\hline $22: 6(n-3)$ & $11.06 \pm 0.6$ & $11.2 \pm 0.46$ & $12.4 \pm 0.3^{*}$ & $11.8 \pm 0.29$ & $22: 6(n-3)$ & $2.04 \pm 0.2^{\#}$ & $0.97 \pm 0.06$ & $0.96 \pm 0.05$ & $1.12 \pm 0.06$ \\
\hline Total SFA & $38.5 \pm 3.2$ & $40.1 \pm 3.3$ & $40.0 \pm 3.3$ & $40.1 \pm 3.3$ & Total SFA & $26.2 \pm 3.9$ & $31.3 \pm 4.8$ & $31.5 \pm 4.9$ & $30.5 \pm 4.6$ \\
\hline Total MUFA & $13.8 \pm 1.5$ & $13.5 \pm 2.0$ & $13.0 \pm 1.9$ & $13.1 \pm 1.9$ & Total MUFA & $39.0 \pm 4.8$ & $47.2 \pm 7.1$ & $48.3 \pm 6.6$ & $48.5 \pm 6.7$ \\
\hline Total PUFA & $47.6 \pm 3.2$ & $46.4 \pm 3.1$ & $47.0 \pm 3.1$ & $46.8 \pm 3.1$ & Total PUFA & $34.8 \pm 3.4^{\#}$ & $21.5 \pm 2.1$ & $20.2 \pm 2.0$ & $21.1 \pm 2.1$ \\
\hline
\end{tabular}

Values represent mean $\pm \mathrm{SEM}, n=7$ for all groups. Means of HF groups not sharing a common letter are statistically significant; $p<0.05$ (ANOVA followed by Tukey test). $* p<0.05, * * p<0.01, * * * p<0.001$ versus LF group (ANOVA followed by Dunnett test; for table clarity to avoid numerous asterisks when all HF groups were different from the LF reference group: ${ }^{*} p<0.05$ LF vs each HF group). HF, high-fat; LF, low-fat; MPL, milk polar lipid; MUFA, monounsaturated fatty acids; PL, phospholipids; PUFA, polyunsaturated fatty acids; SFA, saturated fatty acids; TG, triglycerides.

A

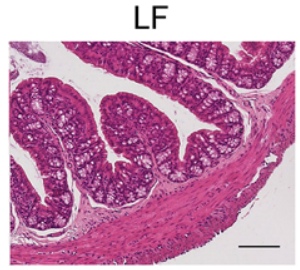

B

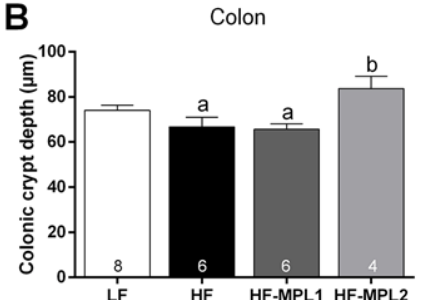

$\mathrm{HF}$

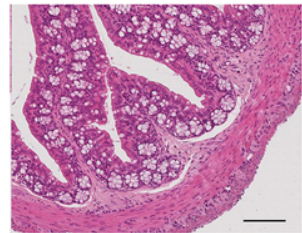

b
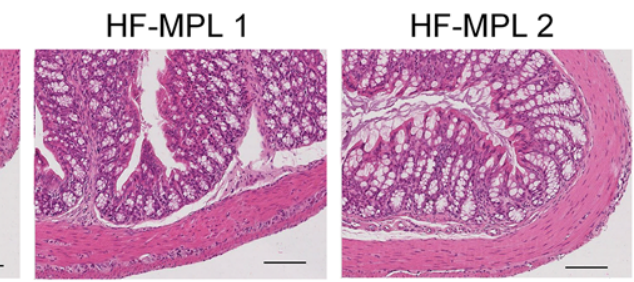

C

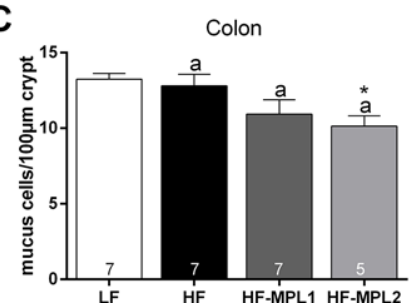

Figure 3. A HF diet supplemented with $1.6 \%$ of MPL increases colonic crypt depth. A) Colon sections stained with hematoxilin and eosin stain, scale bar $=100 \mu \mathrm{m}$. B) Colonic crypt depth and C) Goblet cells were counted as the blue cells stained positive by alcian blue-periodic acid shiff. Data are expressed as mean \pm SEM. To test the impact of MPL amount in the HF diet, data of the three HF groups were analyzed by one-way ANOVA (values not sharing a common letter were significantly different). Additionally, the mean of each HF condition was compared with a LF reference group to evaluate $\mathrm{HF}$ induced alterations ( $* p<0.05$ ANOVA followed by Dunnett's post hoc test vs LF). Number of mice in each group is indicated within the bars. 
precursor of the intestinotrophic peptide GPL-2 (intestinal L cells), was not impacted with MPL feeding (data not shown). There were no differences of expression of antimicrobial proteins such as regenerating islet-derived 3-gamma $(\operatorname{Reg} 3 \gamma)$, phospholipase A2g2 (Pla2g2), and $\alpha$-defensin, which are secreted by Paneth cells to act as a chemical barrier against pathogens and greatly affect the composition of the gut microbiota ${ }^{[30]}$ (data not shown).

\subsection{MPL Supplementation Modulates the Abundance of Bacteria in Cecal Content: Toward a Role of Residual MPL-Derived Lipids in Colon}

HF-MPL1 and HF-MPL2 groups were not significantly different from HF regarding cecum weight $(0.19 \pm 0.01 \mathrm{~g}$ for HF group, $0.18 \pm 0.01 \mathrm{~g}$ for HF-MPL1, and $0.17 \pm 0.01 \mathrm{~g}$ for HF-MPL2). The impact of MPL on gut microbiota was assessed by analyzing the number of total bacteria and some bacteria known for their interesting metabolic effects (Figure 4). The total amount of bacteria and the Firmicutes/Bacteroidetes ratio (Firmicutes, $\approx 60 \%$; Bacteroidetes, $\approx 40 \%$ ) did not differ between groups (results not shown). Of note, the control HF diet did not induce major changes in the studied bacteria versus LF reference group except a higher amount of Bifidobacterium animalis (Figure 4C). However, MPL supplementation induced a shift in the microbial community in comparison with HF group. HF-MPL1 induced an increase in Bifidobacterium spp., in particular B. animalis, versus HF. HF-MPL2 induced a decrease in Lactobacillus reuteri as compared to control HF mice. Moreover, on pooled data of both MPL groups, the abundance of Akkermansia muciniphila was significantly higher in the presence of MPL compared to HF diet devoid of MPL $(p<0.05)$. Significant positive correlations were observed between Bifidobacterium spp. and i) B. animalis ( $r=0.93$; $p<0.0001)$ and ii) Akkermansia muciniphila $(r=0.78 ; p<0.0001$; Figure 4G).

We further explored components that could impact microbiota by analyzing residual dietary lipids in feces (via FA in total lipids) and the composition of bile salts. Compared with HF, MPL supplementation induced in mice feces (Table 4): less myristic acid (C14:0, $p<0.05$ ), less oleic acid (C18:1 (n-9)cis, $p<0.05$ ), and more behenic acid (C22:0, $p<0.001)$, tricosylic acid (C23:0, $p<0.001)$, lignoceric acid (C24:0, $p<0.01)$, and nervonic acid (C24:1, $p<0.01)$. Diets did not differentially impact the overall composition and profile of bile acids nor the ratio of primary (synthesized by the liver) to secondary (synthesized by gut bacteria) bile acids, but MPL2 mice presented less hydrophobic bile acids ( $p<0.0001$; Figure S3, Supporting Information).

We then explored whether gut bacteria abundance were associated with observed differences in fecal lipids and bile salts. There were significant correlations (Figure 4G) between Lactobacillus spp. and i) fecal behenic, tricocylic, lignoceric, and nervonic acids (negative: $r=-0.6 ; p<0.05$ ) and ii) bile salt hydrophobicity (positive: $r=0.52 ; p<0.01$ ). These correlations were also observed with $L$. reuteri ( $r=-0.7 ; p<0.01$ for above-mentioned fecal FA; $r=0.52 ; p<0.01$ for bile salt hydrophobicity). Moreover, bile salt hydrophobicity was also correlated with hepatic gene expression of F4/80 ( $r=0.6, p<0.01)$.

\section{Discussion}

The beneficial impact of MPL and/or milk SM on health is supported by data showing improvement of cholesterol homeostasis and lipid metabolism in rodents in synthetic HF diets. The longterm effects of MPL on macrophage infiltration in liver and adipose tissue, intestinal barrier and microbiota were poorly investigated yet in a more realistic chow-based HF diet. In this study, we show that HF-MPL2 diet resulted in less body weight gain and induced a nonsignificantly lower EAT mass than a HF diet devoid of MPL. We hypothesized that the lower body weight gain is mainly due to the global weight difference of all adipose tissue depots, which could not all be weighted, but we acknowledge this can also be due to potential cumulative weight differences of other organs, bones, and hair. After 10 weeks of a synthetic HF diet supplemented with $0.1 \%$ of milk-SM $(60 \%$ kcal from fat, $0.15 \%$ cholesterol), Norris et al. observed no differences of body weight gain in mice; ${ }^{[21,31]}$ while another synthetic diet for only 4 weeks ( $45 \% \mathrm{kcal}$ from fat) resulted in lower body weight gain when including SM. ${ }^{[20]}$ We previously observed no significant difference in body weight gain and adiposity when $1.2 \%$ MPL was incorporated in a palm-oil-based synthetic HF diet after 8 weeks with an isolipidic design. ${ }^{[23]}$ It thus appears that the efficacy of MPL to lower adiposity is dependent both on the tested dose and on HF diet composition (chow-based versus synthetic, lipid content, and composition). Interestingly, HF-MPL2 diet leads to the lowest adiposity whereas it contains the highest fat level, which will now deserve further calorimetric and energy expenditure experiments to elucidate underlying mechanisms.

Both high-fat fed and ob/ob mice have been associated with a metabolic endotoxemia, dependent on the translocation of LPS notably due to intestinal permeability, that can induce inflammation in target organs, namely adipose tissue and liver. ${ }^{[14,19]}$ Previous studies in mice fed semi-synthetic HF diets (based on starch and casein, $40-60 \% \mathrm{~kJ}$ as fat) reported that addition of milk-SM or MPL decreased HF-induced inflammation, notably in EAT. ${ }^{[21,23,31]}$ Here, the chow-based HF diet treatment itself did not induce inflammation, probably again as it was more balanced and lipids were not composed of palm oil only. Still, HF diet induced increased adiposity. In obesity, macrophage infiltration in white adipose tissue is known to be increased and to induce inflammation of tissues. ${ }^{[32,33]}$ Interestingly, a nonsignificant decrease of macrophage markers F4/80 in liver was observed with the highest MPL dose compared with HF group, and HF-MPL2 was the only group that did not increase liver F4/80 compared to the LF reference group. This can be consistent with a lower gene expression of F4/80 in adipose tissue observed after incorporation of dietary SM in HF diet in mice. ${ }^{[31]}$ However, we did not observe an impact on the gene expression of F4/80 in the adipose tissue nor of the global infiltration marker Cd68. Only Cd11c was increased in EAT after 8 weeks of diet with HF-MPL1. Moreover, the tested diets did not induce systemic low-grade inflammation. Different studies using synthetic HF diets, most often enriched in cholesterol, reported that MPL and/or milk SM decreased hepatic lipid storage. ${ }^{[6,7,20,31]}$ Here, we did not observe differential TG nor PL content in the liver. However, HF-MPL2 induced a higher proportion of very-long-chain FA typical of milk SM in the FA profile of liver PL: whether this could explain a decrease of F4/80 expression would deserve further study. 
A

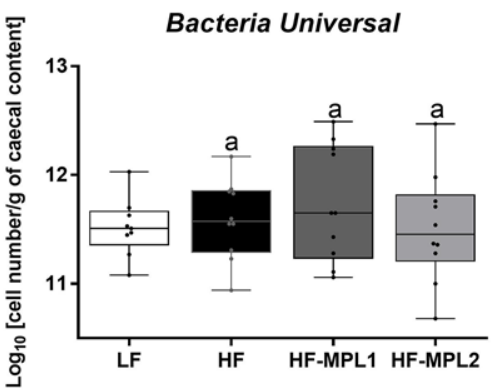

D

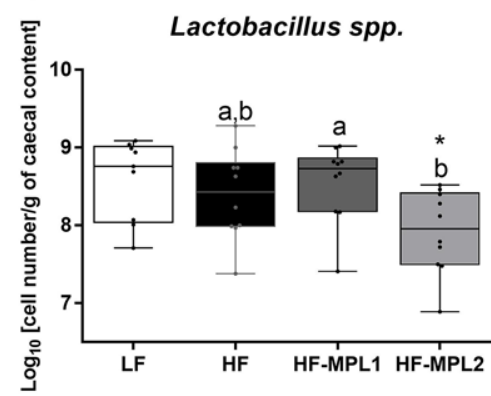

B

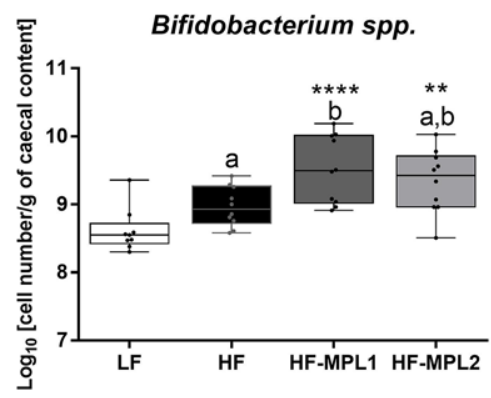

E

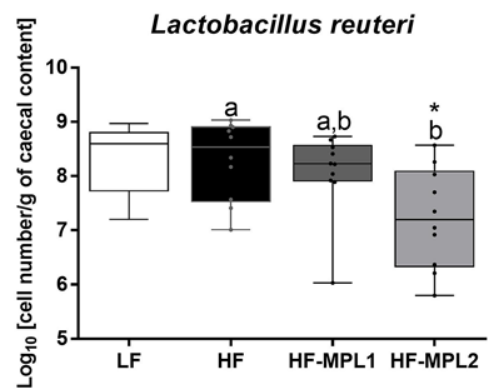

C

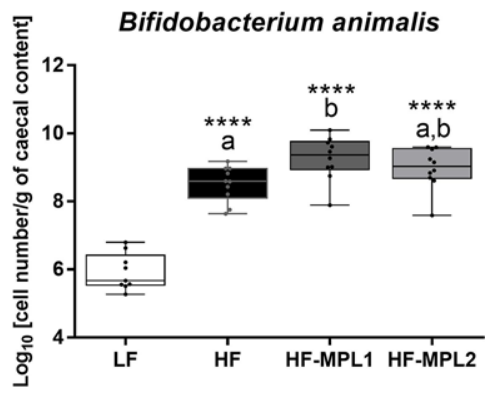

$\mathbf{F}$

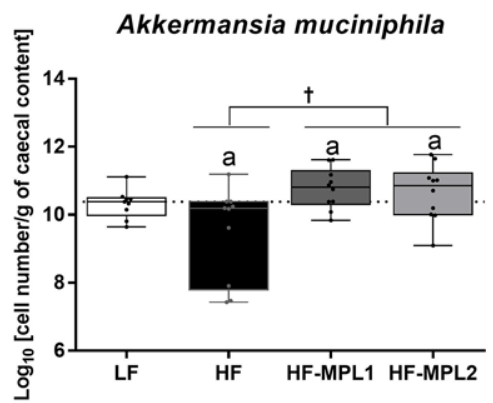

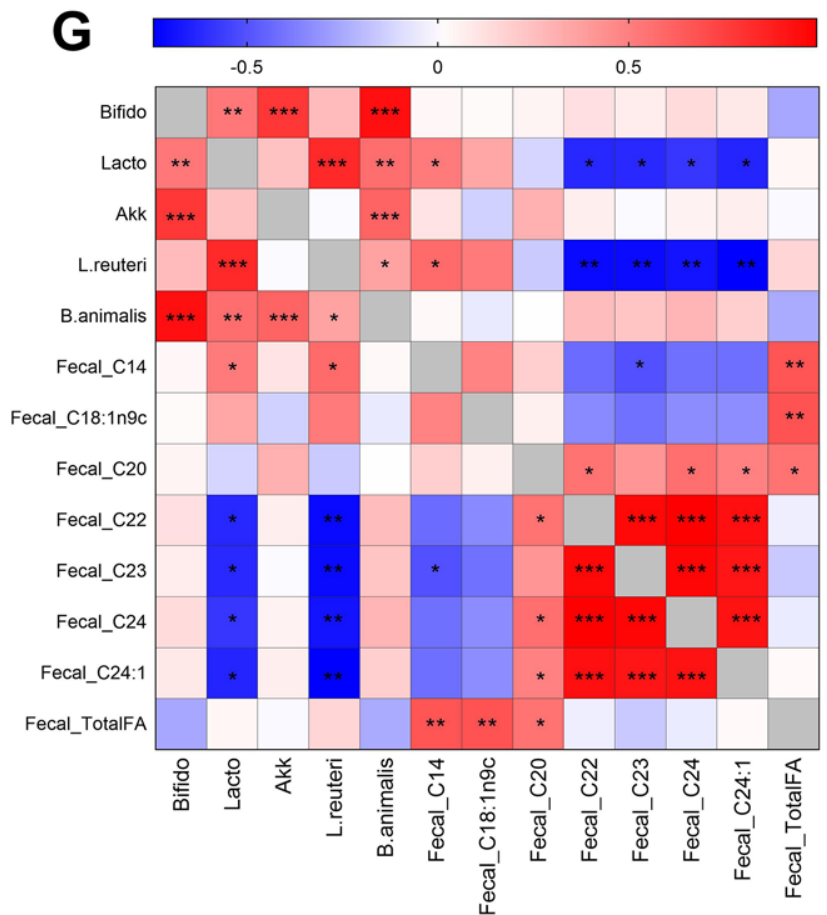

Figure 4. MPL supplementation modulates the abundance of bacteria in the cecal content. A) Total bacteria, B) Bifidobacterium spp., C) Bifidobacterium animalis, D) Lactobacillus spp., E) Lactobacillus reuteri, and F) Akkermansia muciniphila in the cecal content of mice. Data are Whiskers plots with minimum and maximum. To test the impact of MPL amount in the HF diet, data of the three HF groups were analyzed by one-way ANOVA (values not sharing a common letter were significantly different; $\dagger$ for pooled data of HF-MPL1 + HF-MPL2). Additionally, the mean of each HF condition was compared with a LF reference group to evaluate HF induced alterations (" $p<0.05$ ANOVA followed by Dunnett's post hoc test vs LF; $n=10$ mice per group). G) Heat map showing correlations in HF groups. Red represents a positive correlation, white represents a low correlation, and blue represents a negative correlations. Statistical significance was analyzed by Spearman's correlations. *Correlation is significant at the 0.05 level. $* *$ Correlation is significant at the 0.01 level. $* * *$ Correlation is significant at the 0.001 level. 
Table 4. FA composition of mice feces (analyzed in total fecal lipids)

\begin{tabular}{|c|c|c|c|c|}
\hline $\mathrm{FA}\left[\mu \mathrm{g} \mathrm{mg}^{-1}\right.$ feces] & LF & $\mathrm{HF}$ & HF-MPL1 & HF-MPL2 \\
\hline $14: 0$ & $0.22 \pm 0.3^{\#}$ & $0.89 \pm 0.04^{\mathrm{a}}$ & $0.77 \pm 0.03^{\mathrm{a}, \mathrm{b}}$ & $0.71 \pm 0.04^{b}$ \\
\hline $16: 0$ & $2.7 \pm 0.1^{\#}$ & $29.75 \pm 1.84$ & $25.54 \pm 1.22$ & $26.85 \pm 1.88$ \\
\hline $16: 1$ & $0.06 \pm 0.00^{\#}$ & $0.11 \pm 0.01$ & $0.10 \pm 0.00$ & $0.10 \pm 0.00$ \\
\hline 18:0 & $0.52 \pm 0.03^{\#}$ & $3.71 \pm 0.28$ & $3.62 \pm 0.21$ & $4.03 \pm 0.41$ \\
\hline 18:1 $(n-9)$ trans & $0.72 \pm 0.06$ & $1.02 \pm 0.10$ & $1.12 \pm 0.14^{*}$ & $1.17 \pm 0.12^{*}$ \\
\hline 18:1 $(n-9)$ cis & $1.33 \pm 0.09^{\#}$ & $8.64 \pm 0.74^{a}$ & $6.29 \pm 0.39^{b}$ & $6.31 \pm 0.33^{b}$ \\
\hline $18: 1(n-7)$ & $0.21 \pm 0.01^{\#}$ & $0.39 \pm 0.02$ & $0.37 \pm 0.01$ & $0.34 \pm 0.03$ \\
\hline $18: 2(n-6)$ cis & $3.47 \pm 0.31$ & $5.33 \pm 0.43^{\text {*kt }}$ & $4.17 \pm 0.58$ & $3.88 \pm 0.36$ \\
\hline $18: 3(n-3)$ & $0.27 \pm 0.03$ & $0.36 \pm 0.03$ & $0.29 \pm 0.05$ & $0.25 \pm 0.03$ \\
\hline $20: 0$ & $0.07 \pm 0.01^{\#}$ & $0.36 \pm 0.03$ & $0.37 \pm 0.03$ & $0.43 \pm 0.06$ \\
\hline 20:1 & $0.1 \pm 0.01^{\#}$ & $0.20 \pm 0.01$ & $0.17 \pm 0.01$ & $0.18 \pm 0.02$ \\
\hline $22: 0$ & $0.08 \pm 0.01^{\#}$ & $0.15 \pm 0.01^{\mathrm{a}}$ & $0.43 \pm 0.02^{b}$ & $0.61 \pm 0.05^{c}$ \\
\hline $20: 4(n-6)$ & $0.08 \pm 0.01$ & $0.10 \pm 0.02$ & $0.07 \pm 0.01$ & $0.11 \pm 0.03$ \\
\hline 23:0 & $0.02 \pm 0.00$ & $0.04 \pm 0.00^{\mathrm{a}}$ & $0.40 \pm 0.02^{\mathrm{b}, \text {, \#市 }}$ & $0.62 \pm 0.05^{c, k \ldots k}$ \\
\hline $24: 0$ & $0.08 \pm 0.00^{\#}$ & $0.16 \pm 0.01^{\mathrm{a}}$ & $0.43 \pm 0.02^{b}$ & $0.59 \pm 0.05^{c}$ \\
\hline 24:1 & $0.04 \pm 0.00$ & $0.05 \pm 0.00^{\mathrm{a}}$ & $0.07 \pm 0.00^{a^{a, k \times k}}$ & $0.11 \pm 0.01^{b, k \ldots k n}$ \\
\hline Total & $9.95 \pm 0.49^{\#}$ & $51.30 \pm 3.16$ & $44.23 \pm 1.84$ & $46.33 \pm 2.36$ \\
\hline
\end{tabular}

Values represent mean \pm SEM, $n=8$ for LF group, $n=7$ for HF group and $n=5$ for HF-MPL groups. Means of HF groups not sharing a common letter are statistically significant; $p<0.05$ (ANOVA followed by Tukey test). $* p<0.05$, ** $p<0.01$,

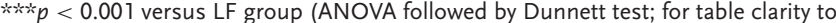
avoid numerous asterisks when all HF groups were different from the LF reference group: ${ }^{\#} p<0.05$ LF vs each HF group). HF, high-fat; LF, low-fat; MPL, milk polar lipid.

The intestinal microbiota appears as a major player in the development of obesity. ${ }^{[34-38]}$ We can note that here the control HF diet did not induce major changes in the studied bacteria versus LF reference group. This lack of alterations may be explained by the presence in our HF diet of chow, which contributes to microbiota homeostasis. Indeed, Dalby et al. have shown that feeding mice a refined diet compared to an unrefined chow-diet induced alterations in gut microbiota. ${ }^{[39]}$ Regarding MPL impact, HFMPL1 significantly increased Bifidobacterium spp., in particular $B$. animalis in mouse cecal content. Cani et al. ${ }^{[40]}$ reported that a decrease of Bifidobacterium spp. was associated with deleterious metabolic outcomes. As here Bifidobacterium spp. was also found correlated with Akkermansia muciniphila, which has otherwise been described to present favorable metabolic effects, ${ }^{[41,42]}$ this suggests an overall prebiotic-like impact of MPL. Moreover, HF-MPL2 mice exhibited a decrease in Lactobacillus spp. as compared to HF-MPL1 group. In comparison with the HF group, HF-MPL2 decreased significantly L. reuteri. We suggest that this could contribute to positive metabolic impact of the highest dose of MPL. Indeed, obese patients present an increased Lactobacillus content $^{[43,44]}$ and increased abundance of $L$. reuteri has been associated with weight gain in some studies, albeit it can also exert protective effects such as anti-inflammatory effects in other cases ${ }^{[45-48]}$ A dietary supplementation with Lactobacillus spp. (L. salivarium and $L$. reuteri) in bird resulted in decreased ileal crypt depth and increased body weight. ${ }^{[49]}$ This is consistent with an hypothesis for a causal link as here we found a decrease of body weight gain, an increase of colonic crypt depth and a decrease in L. reuteri with HF-MPL2. Another study in rat pups also reported increased colonic crypt depth after MFGM supplementation, which was discussed as a favorable impact on gut maturation. ${ }^{[50]}$ However, an increased intestinal crypt depth was also shown to decrease the activities of some enzymes..$^{[51]}$ Whether MPL can modify enzyme activities in the intestine deserves further investigation. We cannot exclude that those morphological changes in the colon may modulate the absorption of bacterial-derivedmetabolites produced locally, which could play a role in the control of inflammation or metabolic disorders in the liver or adipose tissue (like SCFA or conjugated FA). ${ }^{[52,53]}$ Importantly, we ruled out an impact of MPL on the composition of bile salts, which are reported to influence gut physiology and lipid metabolism. ${ }^{[54,55]}$ However, the mechanisms by which HF-MPL2 led to less hydrophobic bile acids will deserve further investigation. Moreover, mucus layer is an important component of the colonic physiology and we previously observed that MPL in a synthetic HF diet increased the number of colonic goblet cells. ${ }^{[23]}$ Here, mucus cells appeared unaffected by MPL addition in the chow-based HF-diet suggesting an additional impact of background diet composition.

Among the PL in milk, SM is of specific interest because of its previously reported beneficial effects in rodents. Here MPLenriched diets contained $0.25 \%$ of SM (HF-MPL1) or $0.38 \%$ of SM (HF-MPL2). The effects of MPL on decreased cholesterol absorption $^{[7]}$ and inhibition of colonic tumor ${ }^{[9]}$ in rodents have also been observed using diets with purified milk SM at concentrations as low as $0.1 \%,{ }^{[10,11,56]}$ suggesting that effects seen with MPL may be at least partly due to SM. The least weight gain observed with MPL and the higher content in Bifidobacteria could be due to the specific effect of milk-SM because a HF diet supplemented with $0.25 \%$ of SM showed similar result. ${ }^{[2]}$ Dietary SM, for example from MPL, is not completely digested and absorbed in the small intestine, so that residues can reach the colon. ${ }^{[57,58]}$ Here, we reveal a correlation between the fecal loss of FA specific of milk SM and the cecal abundance of Lactobacillus. We, therefore, suggest a causal impact of unabsorbed SM-derived lipids in colon on gut bacteria and possibly on colonic physiology considering crypt depth impact, which should now be causally demonstrated. We also observed a lower vaccenic acid proportion in liver lipids in MPL2 group. Although our analysis did not discriminate cis- and trans-isomers of vaccenic acid, we can note that transvaccenic acid is mainly produced from bacterial metabolism. ${ }^{[59]}$ As such, it is naturally contained in milk due to ruminal fermentation, together with rumenic acid (18:2 cis- 9 trans-11 CLA). Notably, Lactobacillus spp. of the gut microbiota have also been described to be involved in the local production of CLA acid in the intestine. ${ }^{[6]}$ Here, we propose as a further research perspective that MPL2 effect on gut Lactobacillus spp. could have impacted liver metabolism, as suggested by the content of liver PL in both SM-specific FA and vaccenic acid.

A limitation of our study is a common issue in nutrition research: as one lipid ingredient (here MPL) is added in a diet additionally to the other lipids, we cannot rule out that some effects can be due to the concomitant decrease of some other dietary components of the chow including proteins, starch and some micronutrients notably. However, a major strength of our study is that we chose to use the least favorable design to test the impact of MPL, by including them in the diet additionally to palm oil. If we had removed part of palm oil when adding MPL with an 
aim to keep total lipids constant, such lower palm oil content (in a chow-based diet that also contains a wide variety of beneficial ingredients) could have induced favorable effects, which would have biased conclusions on the real MPL impact. We think our choice of increased dietary lipid content when adding MPL finally strengthens our conclusions that adding the MPL-rich lipid extract improved metabolism, despite a concomitant slightly decreased proportion of chow ingredients in the MPL-containing diet compared with the control HF diet. Furthermore, previous studies on MPL have tested their effects in the context of purified diets, which do not emulate human diets, whereas here we used a complex high-fat chow diet. This is a novel aspect of the present study of metabolic importance, as refined low-fat (purified) diets are known to induce differences in gut microbiome (e.g., increased the Firmicutes-to-Bacteroidetes ratio and reduced diversity) unlike unrefined chow diets. ${ }^{[39]}$

In summary, here we characterized in $\mathrm{C} 57 \mathrm{Bl} / 6$ mice the influence of different amounts of MPL $(1.1 \%$ or $1.6 \%)$ added in a chow-based HF diet, on body weight gain, intestinal physiology, and gut microbiota. We demonstrated that despite a higher fat content, HF-MPL2 led to the lowest body weight gain and did not induce metabolic inflammation, while HF-MPL1 did not prevent weight gain. Moreover, we suggest that a limited adiposity in HFMPL2 may have contributed to this effect. A differential impact of the two MPL doses was observed on the gut microbiota and colon physiology, as HF-MPL1 increased Bifidobacteria with no impact on colonic crypt depth, while HF-MPL2 decreased Lactobacillus and increased colonic crypt depth. Of note, as no correlation was observed between gut bacteria and anthropometric and metabolic parameters, we cannot conclude on a direct impact of gut bacteria. However, a very significant result was the dose-response increase in fecal loss of FA specific of milk SM. Therefore, among possible mechanisms that can impact the distal gut and the microbiota, we suggest the role of a threshold level of residual lipids specific of MPL reaching the colon. MPL are highly bioactive components because they contain sphingolipids and notably SM that can contribute to the observed effects. When developing strategies for enhancing gut physiology for favorable metabolic impacts using bioactive food ingredients, it appears important to consider the interactions between the different components of the diet and of the intestinal barrier. Future research shall deeper evaluate and understand the properties of milk SM as a compound involved in the beneficial effects of MPL and in gut physiology, which could present interesting effects in obesity or in leaky gut diseases.

\section{Supporting Information}

Supporting Information is available from the Wiley Online Library or from the author.

\section{Acknowledgements}

M.M., F.L., and M.-C.M. designed the research; M.M. and M.-C.M. wrote the manuscript; M.M., F.L., A.D, C.B, E.M., E.L., C.L.-P., V.S., L.G., S.V., K.B., F.J., D.C., L.H., D.R., P.P., L.B., A.N., N.D., and M.-C.M. conducted the research; M.M. and M.-C.M. performed statistical analysis; A.N., L.B., and N.D. were associated with scientific expertise, supervised research on mi- crobiota and gut/liver histology, and were involved in discussion of results, and review of the manuscript. A.D. and C.B. provided technical support for the analysis; C.B. provided support for in vivo experiments; E.M. and E.L. performed $\mathrm{qPCR}$ analyses. F.J. provided essential material and performed lipid analysis. K.B., F.J., C.L.-P., V.S., and D.C performed lipid analyses. P.P. contributed to histology; L.H. and D.R. performed bile salt analysis; L.G. and S.V. performed plasma biochemical analysis of inflammatory markers using Luminex. V.S., D.R., F.L., L.B., A.N., and N.D. reviewed the manuscript. All authors read and approved the final manuscript. M.C.M. takes the final responsibility for the manuscript. Marine Milard acknowledges a PhD grant from French Ministry for Higher Education and Research via EDISS and Université Claude Bernard Lyon 1. The authors acknowledge CNIEL (French Dairy Interbranch Organization) for funding the study. The authors thank Corinne Marmonier (CNIEL) for useful comments on the manuscript. The authors additionally thank Constance Boyer and Benjamin Choque (CNIEL) and Sébastien Serieye (SodiaalCandia R\&D), Nikta Vaghefi (Savencia), Chalotte Baudry (Lactalis), and Renaud Mesdagh (Bongrain/Soredab) for fruitful discussions. The authors thank Sophie Thenet (INSERM UMR S1138, France), Agnes Girard-Egrot (ICBMS, Villeurbanne, France), and Corinne Malpuech-Brugère (Human Nutrition Unit, INRA-Université d'Auvergne, Clermont-Ferrand, France) for critical discussions on the results. The authors thank CellImaP (Plateforme d'imagerie cellulaire, Federative structure for research 100, Dijon, France) for facilities in histological technics and Dominique Martel, Sylvain Tuller and Cyril Campenon for diet formulation. Richard Tallon is acknowledged for providing MPL ingredient. Sébastien Serieye is acknowledged for providing anhydrous milk fat. The authors thank Armelle Penhoat for fruitful discussions. The authors thank Bouazza Es Saadi, Isabelle Blave, and Adeline Cestre for skillful technical assistance, and Loïc Damon and Chloé Robert for their help during animal study. Carole Knibbe is acknowledged for her assistance in completing statistical analysis design.

\section{Conflict of Interest}

This study was funded by CNIEL (French Dairy Interbranch Organization) (SMILE project). M.-C.M. received other research fundings on other topics from Sodiaal-Candia R\&D and Nutricia Research and has consultancy activities for food and dairy companies. These activities had no link with the present study. M.-C.M. coordinated a research project funded by the $\mathrm{Na}$ tional Research Agency on the valuing and health properties of MPL (VALOBAB ANR-2011-0007, 2012-2016). F.J. is an employee of ITERG (Fats, oils and crops Interbranch Technical Institute). F.L. and M.M. participated in a research project on another topic funded by Sodiaal-Candia R\&D. P.P. received research funding on other topics from Ingredia. N.M.D. is a recipient of grants from FRS-FNRS, from Wallonia supported by the competitive cluster Wagralim (ADIPOSTOP project, convention 7366; FOOD4GUT project, convention 1318148) and from the European Union's Seventh Framework Program (MYNEWGUT project, grant agreement no. 613979). Other authors have declared no conflict of interest.

\section{Keywords}

adiposity, bile salts, feces, gut barrier, microbiota, milk fat globule membrane, phospholipids, sphingomyelin

Received: October 8, 2018

Revised: December 18, 2018

Published online: January 25, 2019

[1] H. Mu, C. E. Hoy, Prog. Lipid Res. 2004, 43, 105.

[2] M. C. Michalski, C. Genot, C. Gayet, C. Lopez, F. Fine, F. Joffre, J. L. Vendeuvre, J. Bouvier, J. M. Chardigny, K. Raynal-Ljutovac, Prog. Lipid Res. 2013, 52, 354. 
[3] V. L. Spitsberg, J. Dairy Sci. 2005, 88, 2289.

[4] H. Vesper, E. M. Schmelz, M. N. Nikolova-Karakashian, D. L. Dillehay, D. V. Lynch, A. H. Merrill, Jr., J. Nutr. 1999, 129, 1239.

[5] J. S. Cohn, E. Wat, A. Kamili, S. Tandy, Curr. Opin. Lipidol. 2008, 19, 257.

[6] E. Wat, S. Tandy, E. Kapera, A. Kamili, R. W. Chung, A. Brown, M. Rowney, J. S. Cohn, Atherosclerosis 2009, 205, 144.

[7] A. Kamili, E. Wat, R. W. Chung, S. Tandy, J. M. Weir, P. J. Meikle, J. S. Cohn, Nutr. Metab. 2010, 7, 90

[8] M. Lecomte, C. Bourlieu, E. Meugnier, A. Penhoat, D. Cheillan, G. Pineau, E. Loizon, M. Trauchessec, M. Claude, O. Menard, A. Geloen, F. Laugerette, M. C. Michalski, J. Nutr. 2015, 145, 1770.

[9] D. R. Snow, R. Jimenez-Flores, R. E. Ward, J. Cambell, M. J. Young, I. Nemere, K. J. Hintze, J. Agric. Food Chem. 2010, 58, 2157.

[10] D. L. Dillehay, S. K. Webb, E. M. Schmelz, A. H. Merrill, Jr., J. Nutr. 1994, 124, 615

[11] E. M. Schmelz, D. L. Dillehay, S. K. Webb, A. Reiter, J. Adams, A. H. Merrill, Jr., Cancer Res 1996, 56, 4936.

[12] T. Sawai, R. A. Drongowski, R. W. Lampman, A. G. Coran, C. M. Harmon, Pediatr. Surg. Int. 2001, 17, 269.

[13] A. P. Moreira, T. F. Texeira, A. B. Ferreira, C. Peluzio Mdo, C. Alfenas Rde, Br. J. Nutr. 2012, 108, 801.

[14] P. D. Cani, R. Bibiloni, C. Knauf, A. Waget, A. M. Neyrinck, N. M. Delzenne, R. Burcelin, Diabetes 2008, 57, 1470

[15] Y. Y. Lam, C. W. Ha, C. R. Campbell, A. J. Mitchell, A. Dinudom, J. Oscarsson, D. I. Cook, N. H. Hunt, I. D. Caterson, A. J. Holmes, L. H. Storlien, PLoS One 2012, 7, e34233.

[16] C. W. Teshima, L. A. Dieleman, J. B. Meddings, Ann. N. Y. Acad. Sci. 2012, 1258, 159

[17] A. P. Kerckhoffs, L. M. Akkermans, M. B. de Smet, M. G. Besselink, F. Hietbrink, I. H. Bartelink, W. B. Busschers, M. Samsom, W. Renooij, Dig. Dis. Sci. 2010, 55, 716.

[18] R. Burcelin, V. Crivelli, A. Dacosta, A. Roy-Tirelli, B. Thorens, Am. J. Physiol. Endocrinol. Metabol. 2002, 282, E834.

[19] F. Laugerette, C. Vors, A. Geloen, M. A. Chauvin, C. Soulage, S. Lambert-Porcheron, N. Peretti, M. Alligier, R. Burcelin, M. Laville, H. Vidal, M. C. Michalski, J. Nutr. Biochem. 2011, 22, 53.

[20] G. H. Norris, C. Jiang, J. Ryan, C. M. Porter, C. N. Blesso, J. Nutr. Biochem. 2016, 30, 93.

[21] G. Norris, C. Porter, C. Jiang, C. Blesso, Beverages 2017, 3, 37.

[22] C. Bourlieu, D. Cheillan, M. Blot, P. Daira, M. Trauchessec, S. Ruet, J. Y. Gassi, E. Beaucher, B. Robert, N. Leconte, S. Bouhallab, F. Gaucheron, G. Gesan-Guiziou, M. C. Michalski, Food Chem. 2018, 240, 67.

[23] M. Lecomte, L. Couedelo, E. Meugnier, P. Plaisancie, M. Letisse, B. Benoit, L. Gabert, A. Penhoat, A. Durand, G. Pineau, F. Joffre, A. Geloen, C. Vaysse, F. Laugerette, M. C. Michalski, Mol. Nutr. Food Res. 2016, 60, 609.

[24] Research CfDEa, e., Guidance for industry: estimating themaximum safe starting dose in initial clinical trials for therapeutics in adult healthy volunteers., 2005.

[25] F. Laugerette, G. Pineau, C. Vors, M. C. Michalski, J. Anal. Bioanal. Tech. 2015, 6:251.

[26] J. Folch, M. Lees, G. H. Sloane Stanley, J. Biol. Chem. 1957, 226, 497.

[27] L. Gabert, C. Vors, C. Louche-Pelissier, V. Sauvinet, S. LambertPorcheron, J. Drai, M. Laville, M. Desage, M. C. Michalski, Rapid Commun. Mass Spectrom. 2011, 25, 2697.

[28] L. B. Bindels, A. M. Neyrinck, S. P. Claus, C. I. Le Roy, C. Grangette, B. Pot, I. Martinez, J. Walter, P. D. Cani, N. M. Delzenne, ISME J. 2016, 10, 1456.

[29] L. Humbert, M. A. Maubert, C. Wolf, H. Duboc, M. Mahe, D. Farabos, P. Seksik, J. M. Mallet, G. Trugnan, J. Masliah, D. Rainteau, J. Chromatogr. B 2012, 899, 135.
[30] C. L. Bevins, N. H. Salzman, Nat. Rev. Microbiol. 2011, 9, 356.

[31] G. H. Norris, C. M. Porter, C. Jiang, C. L. Millar, C. N. Blesso, J. Nutr. Biochem. 2017, 40, 36.

[32] H. Xu, G. T. Barnes, Q. Yang, G. Tan, D. Yang, C. J. Chou, J. Sole, A Nichols, J. S. Ross, L. A. Tartaglia, H. Chen, J. Clin. Invest. 2003, 112, 1821.

[33] S. P. Weisberg, D. McCann, M. Desai, M. Rosenbaum, R. L. Leibel, A W. Ferrante, Jr., J. Clin. Invest. 2003, 112, 1796.

[34] I. T. Harley, C. L. Karp, Mol. Metab. 2012, 1, 21.

[35] R. E. Ley, F. Backhed, P. Turnbaugh, C. A. Lozupone, R. D. Knight, J. I. Gordon, Proc. Natl. Acad. Sci. USA 2005, 102, 11070.

[36] P. J. Turnbaugh, M. Hamady, T. Yatsunenko, B. L. Cantarel, A. Duncan, R. E. Ley, M. L. Sogin, W. J. Jones, B. A. Roe, J. P. Affourtit, M. Egholm, B. Henrissat, A. C. Heath, R. Knight, J. I. Gordon, Nature 2009, 457, 480.

[37] R. E. Ley, P. J. Turnbaugh, S. Klein, J. I. Gordon, Nature 2006, 444, 1022.

[38] P. J. Turnbaugh, R. E. Ley, M. A. Mahowald, V. Magrini, E. R. Mardis, J. I. Gordon, Nature 2006, 444, 1027.

[39] M. J. Dalby, A. W. Ross, A. W. Walker, P. J. Morgan, Cell Rep. 2017, 21, 1521.

[40] P. D. Cani, A. M. Neyrinck, F. Fava, C. Knauf, R. G. Burcelin, K. M Tuohy, G. R. Gibson, N. M. Delzenne, Diabetologia 2007, 50, 2374.

[41] C. H. Hansen, L. Krych, D. S. Nielsen, F. K. Vogensen, L. H. Hansen, S. J. Sorensen, K. Buschard, A. K. Hansen, Diabetologia 2012, 55, 2285.

[42] M. Schneeberger, A. Everard, A. G. Gomez-Valades, S. Matamoros, S. Ramirez, N. M. Delzenne, R. Gomis, M. Claret, P. D. Cani, Sci. Rep. 2015, 5, 16643

[43] F. Armougom, M. Henry, B. Vialettes, D. Raccah, D. Raoult, PLoS One 2009, 4, e7125

[44] J. Stsepetova, E. Sepp, H. Kolk, K. Loivukene, E. Songisepp, M. Mikelsaar, Br.J. Nutr. 2011, 105, 1235.

[45] M. Million, M. Maraninchi, M. Henry, F. Armougom, H. Richet, P. Carrieri, R. Valero, D. Raccah, B. Vialettes, D. Raoult, Int.J. Obes. 2012, $36,817$.

[46] M. Million, E. Angelakis, M. Paul, F. Armougom, L. Leibovici, D. Raoult, Microb. Pathog. 2012, 53, 100.

[47] R. Luoto, M. Kalliomaki, K. Laitinen, E. Isolauri, Int. J. Obes. 2010, 34 , 1531.

[48] Y. Kadooka, M. Sato, K. Imaizumi, A. Ogawa, K. Ikuyama, Y. Akai, M. Okano, M. Kagoshima, T. Tsuchida, Eur. J. Clin. Nutr. 2010, 64, 636.

[49] W. A. Awad, K. Ghareeb, S. Abdel-Raheem, J. Bohm, Poult. Sci. 2009, 88,49

[50] G. Bhinder, J. M. Allaire, C. Garcia, J. T. Lau, J. M. Chan, N. R. Ryz, E. S. Bosman, F. A. Graef, S. M. Crowley, L. S. Celiberto, J. C. Berkmann, R. A. Dyer, K. Jacobson, M. G. Surette, S. M. Innis, B. A. Vallance, Sci. Rep. 2017, 7, 45274.

[51] D. J. Hampson, Res. Vet. Sci. 1986, 40, 32.

[52] G. den Besten, K. van Eunen, A. K. Groen, K. Venema, D. J. Reijngoud, B. M. Bakker, J. Lipid Res. 2013, 54, 2325.

[53] W. Scheppach, Gut 1994, 35, S35.

[54] M. Schonewille, J. F. de Boer, A. K. Groen, Curr. Opin. Lipidol. 2016, 27, 295.

[55] O. Martinez-Augustin, F. Sanchez de Medina, World J. Gastroenterol. 2008, 14, 5630

[56] E. R. M. Eckhardt, D. Q. H. Wang, J. M. Donovan, M. C. Carey, Gastroenterology 2002, 122, 948

[57] Å. Nilsson, R.-D. Duan, Chem. Phys. Lipids 1999, 102, 97.

[58] A. Nilsson, Biochim. Biophys. Acta 1968, 164, 575

[59] C. Druart, A. M. Neyrinck, B. Vlaeminck, V. Fievez, P. D. Cani, N. M Delzenne, PLoS One 2014, 9, e87560.

[60] H. Y. Lee, J. H. Park, S. H. Seok, M. W. Baek, D. J. Kim, K. E. Lee, K. S Paek, Y. Lee, J. H. Park, Biochim. Biophys. Acta 2006, 1761, 736. 